\title{
Renal immune surveillance and dipeptidase-1 contribute to contrast-induced acute kidney injury
}

\author{
Arthur Lau, ${ }^{1,2}$ Hyunjae Chung, 1,2 Takanori Komada, ${ }^{1,2}$ Jaye M. Platnich, ${ }^{1,2}$ Christina F. Sandall, ${ }^{3,4}$ Saurav Roy Choudhury, ${ }^{5,6}$ \\ Justin Chun,, ${ }^{1,2}$ Victor Naumenko,, 2,7 Bas G.J. Surewaard, 2,7 Michelle C. Nelson, ${ }^{1,2}$ Annegret Ulke-Lemée, ${ }^{3,4}$ Paul L. Beck,, \\ Hallgrimur Benediktsson, ${ }^{2,8}$ Anthony M. Jevnikar, ${ }^{9}$ Sarah L. Snelgrove, ${ }^{10}$ Michael J. Hickey, ${ }^{10}$ Donna L. Senger, ${ }^{5,6}$ \\ Matthew T. James, ${ }^{1,4}$ Justin A. Macdonald, ${ }^{3,4}$ Paul Kubes, ${ }^{2,7}$ Craig N. Jenne, ${ }^{2,7}$ and Daniel A. Muruve ${ }^{1,2}$ \\ 1Department of Medicine, ${ }^{2}$ Snyder Institute for Chronic Diseases, ${ }^{3}$ Department of Biochemistry and Molecular Biology, ${ }^{4}$ Libin Cardiovascular Institute of Alberta, ${ }^{5}$ Department of Oncology, ${ }^{6}$ Arnie \\ Charbonneau Cancer Institute, 'Department of Microbiology, Immunology, and Infectious Diseases, and ${ }^{8}$ Department of Pathology and Laboratory Medicine, University of Calgary, Calgary, Alberta, \\ Canada. ${ }^{9}$ Department of Medicine, University of Western Ontario, London, Ontario, Canada. ${ }^{10}$ Centre for Inflammatory Diseases, Monash University Department of Medicine, Monash Medical Centre, \\ Clayton, Victoria, Australia.
}

Radiographic contrast agents cause acute kidney injury (AKI), yet the underlying pathogenesis is poorly understood. Nod-like receptor pyrin containing 3-deficient (NIrp3-deficient) mice displayed reduced epithelial cell injury and inflammation in the kidney in a model of contrast-induced AKI (CI-AKI). Unexpectedly, contrast agents directly induced tubular epithelial cell death in vitro that was not dependent on NIrp3. Rather, contrast agents activated the canonical Nlrp3 inflammasome in macrophages. Intravital microscopy revealed diatrizoate (DTA) uptake within minutes in perivascular $\mathrm{CX}_{3} \mathrm{CR}^{+}$resident phagocytes in the kidney. Following rapid filtration into the tubular luminal space, DTA was reabsorbed and concentrated in tubular epithelial cells via the brush border enzyme dipeptidase-1 in volumedepleted but not euvolemic mice. LysM-GFP+ macrophages recruited to the kidney interstitial space ingested contrast material transported from the urine via direct interactions with tubules. $\mathrm{Cl}-\mathrm{AKI}$ was dependent on resident renal phagocytes, IL-1, leukocyte recruitment, and dipeptidase-1. Levels of the inflammasome-related urinary biomarkers IL-18 and caspase- 1 were increased immediately following contrast administration in patients undergoing coronary angiography, consistent with the acute renal effects observed in mice. Taken together, these data show that $\mathrm{Cl}-\mathrm{AKI}$ is a multistep process that involves immune surveillance by resident and infiltrating renal phagocytes, Nlrp3-dependent inflammation, and the tubular reabsorption of contrast via dipeptidase-1.

\section{Introduction}

Contrast-induced acute kidney injury (CI-AKI) is caused by iodinated contrast media for diagnostic imaging (1). Most patients with normal kidney function who receive contrast will not experience any renal complications; however, those with volume depletion or chronic kidney disease (CKD) are at increased risk for CI-AKI (2-4). CI-AKI occurs within 24-72 hours following the administration of radiographic contrast agents and is associated with adverse outcomes including acute renal failure requiring dialysis, worsening of $\mathrm{CKD}$, cardiovascular events, and increased health care costs (2). Although numerous clinical and epidemiological studies have characterized the risk factors and incidence rates of CI-AKI in stratified patient populations, the underlying pathogenesis of CI-AKI is poorly understood $(1,5)$.

\section{Related Commentary: p. 2754}

Conflict of interest: DAM is cofounder of and has an equity position in Arch Biopartners Inc. PLB, SRC, DLS, JAM, and PK have equity positions in Arch Biopartners Inc. A patent application for dipeptidase-1 inhibition in acute kidney injury is pending.

Submitted: August 1, 2017; Accepted: April 10, 2018.

Reference information: J Clin Invest. 2018;128(7):2894-2913.

https://doi.org/10.1172/JCI96640.
Contrast agents are known to induce a variety of alterations in the kidney that include medullary ischemia, formation of reactive oxygen species, and tubular cell toxicity (1); however, the exact mechanism of tissue injury, and specifically the role of inflammation, has not been clearly characterized. The incomplete understanding of CI-AKI has hampered the development of new therapeutic strategies to prevent or minimize CI-AKI.

Inflammation is an integral component of acute and CKDs. Leukocytes play important roles in kidney parenchymal injury, repair, and fibrosis $(6,7)$. The kidney contains an intrinsic immune surveillance system that consists of resident macrophages and dendritic cells (herein referred to as resident renal phagocytes) expressing a series of overlapping markers such as CD11b, CD11c, $\mathrm{F} 4 / 80$, and $\mathrm{CX}_{3} \mathrm{CR} 1$ (8). The current understanding of the intrarenal immune system in kidney disease remains limited and is inferred primarily from cell depletion studies (7). While kidneyresident renal phagocytes contribute to the pathogenesis of kidney disease, the respective temporal behaviors, cell-cell interactions, and specific role of various leukocyte populations in renal pathophysiology are not fully characterized. Furthermore, the additional contribution of leukocyte populations recruited from the systemic circulation in propagating kidney injury and inflammation requires ongoing clarification. 
A
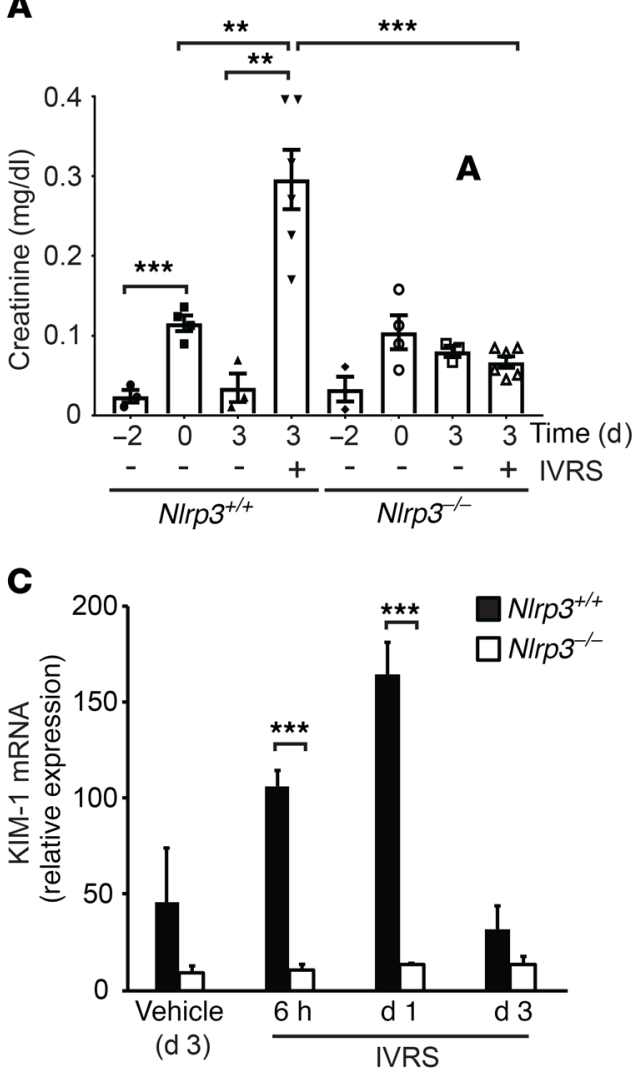

$\mathbf{E}$

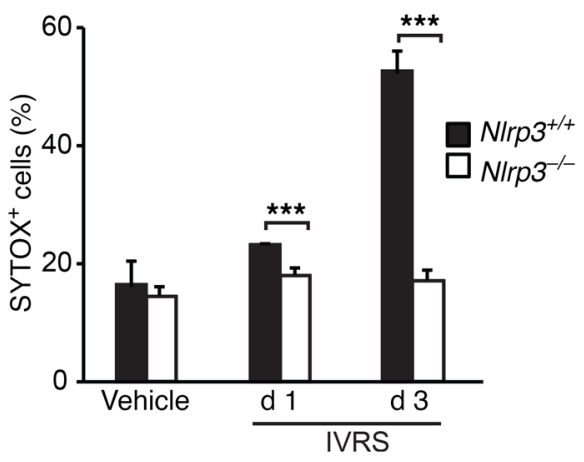

B

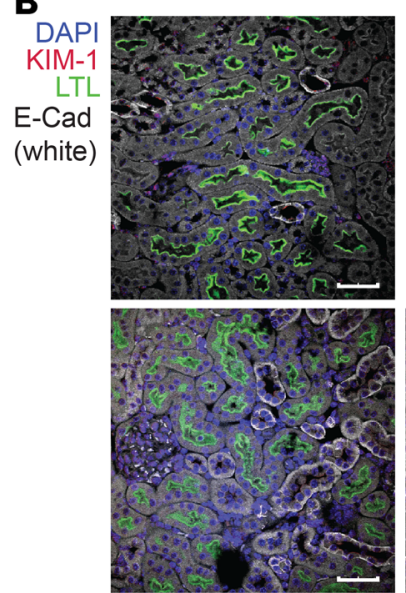

Vehicle

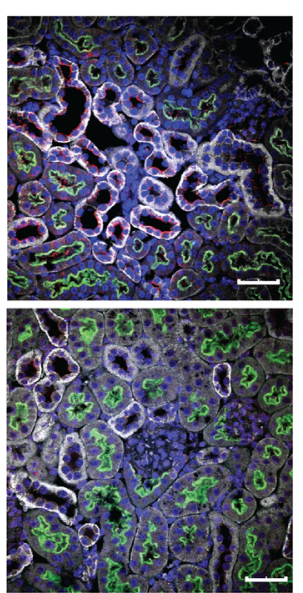

d 1

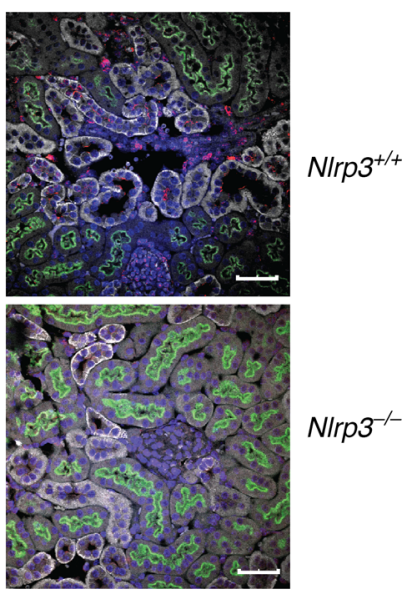

d 3
IVRS

D

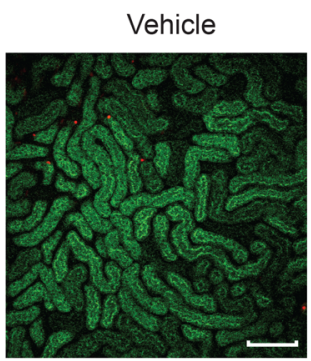

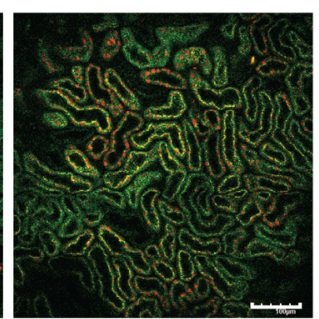

$\mathrm{NIrp3}^{+/+}$
IVRS
Figure 1. Cl-AKI is dependent on NIrp3. WT $\left(\mathrm{NIrp3}^{+/+}\right)$and $\mathrm{NIrp3}^{-/-}$mice were treated with vehicle control or ioversol (IVRS) and assessed at 1-3 days. (A) Renal function as determined by serum creatinine (day $3, \mathrm{Nlrp3}^{+/+}$vs. N/rp3 $3^{-/,},{ }^{* *} P=0.0001, n=6 /$ group, ANOVA). Volume depletion demonstrated an effect on serum creatinine (ref. values $0.04-0.08 \mathrm{mg} / \mathrm{dl})\left(\mathrm{NIrp3}^{+/+}\right.$, day -2 vs. day $0,{ }^{* *} P=0.001, n=3-4$ /group, ANOVA), but it returned to baseline on day 3 (NIrp3 ${ }^{+/+}$day 3 , no IVRS vs. IVRS, ${ }^{* *} P=0.0023, n=3-6 /$ group, ANOVA). (B) Fixed kidney tissue was analyzed for tissue injury using KIM-1 (red). Images were taken with a fluorescence confocal microscope. Labels: nuclei, DAPI (blue); tubules, LTL (green); E-cadherin (E-Cad; white); KIM-1 (red). Image is representative of 3 independent experiments. Scale bars: $50 \mu \mathrm{m}$. (C) Real-time PCR for KIM-1 mRNA expression (NIrp3 ${ }^{+/+}$vs. NIrp3 $3^{-/}, 6$ hours: ${ }^{* * *} P=0.0005$, day $1:{ }^{* * *} P=$ $0.001, n=3-5$ /group, ANOVA). (D) Cellular injury in $\mathrm{NIrp3}^{+/+}$and $\mathrm{NIrp3}^{-/-}$mice determined by multiphoton intravital microscopy and SYTOX positivity of kidney TECs. Image is representative of 3 independent experiments. Scale bars: $200 \mu \mathrm{m}$. (E) Quantitation of SYTOX-positive cells at indicated time points ( $\mathrm{NIrp3}^{+/+}$vs. NIrp3 ${ }^{-1-}$, day 1 : ${ }^{* *} P=0.001$, day 3 : ${ }^{* *} P<0.001$, $n=7-15$ (group, ANOVA).
Recently, several studies employed multiphoton intravital microscopy to demonstrate essential roles for resident renal phagocytes positioned adjacent to peritubular capillaries in the immune surveillance of circulating immune complexes $(9,10)$. The resident renal phagocytes were essential to initiate immune responses, although the exact innate molecular pathways activated within these cells were not elucidated. Furthermore, to our knowledge, the contribution of tubular cells to immune surveillance of molecules filtered and reabsorbed from the urine has never been explored. The role of immune surveillance in response to toxininduced kidney injury such as CI-AKI has also not been examined.

Nod-like receptor pyrin containing 3 (Nlrp3) is an innate pattern recognition receptor and important immune sensor that has canonical and noncanonical roles in models of kidney disease (1115). The canonical Nlrp3 inflammasome is a platform for caspase-1 activation that regulates the maturation of IL-1 $\beta$ and IL-18. Studies from our laboratory show that within the epithelial cell compartment of the kidney, Nlrp3 function is primarily noncanonical, regulating a platform for caspase-8 activation and apoptotic cell death rather than caspase-1 and cytokine maturation (11). Thus, the canonical Nlrp3 inflammasome likely resides primarily within resident renal phagocytes or circulating leukocytes recruited to the injured kidney (16).

In this study, we show that CI-AKI is a multistep process involving immune activation coordinated by resident renal phagocytes, the tubular reabsorption of contrast media, and recruited leuko- 


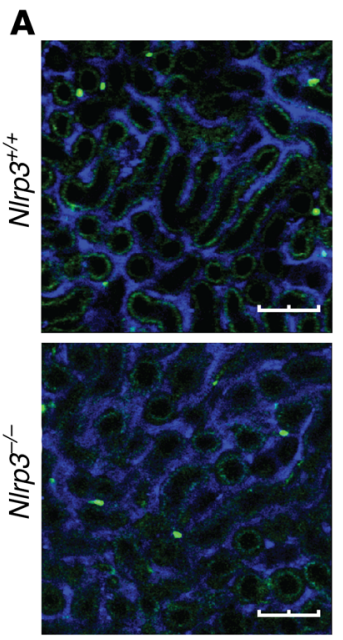

Vehicle
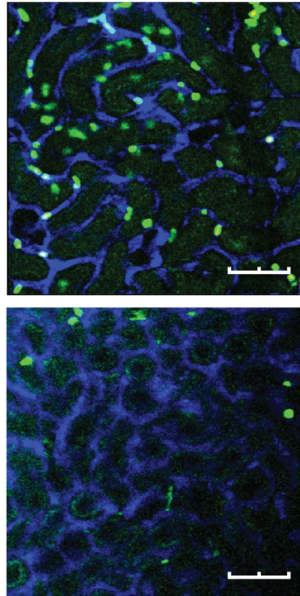

$6 \mathrm{~h}$

C

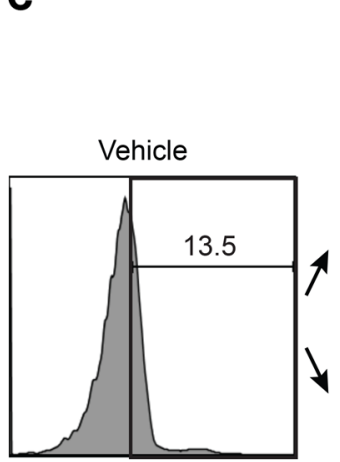

LysM-GFP $^{+}$

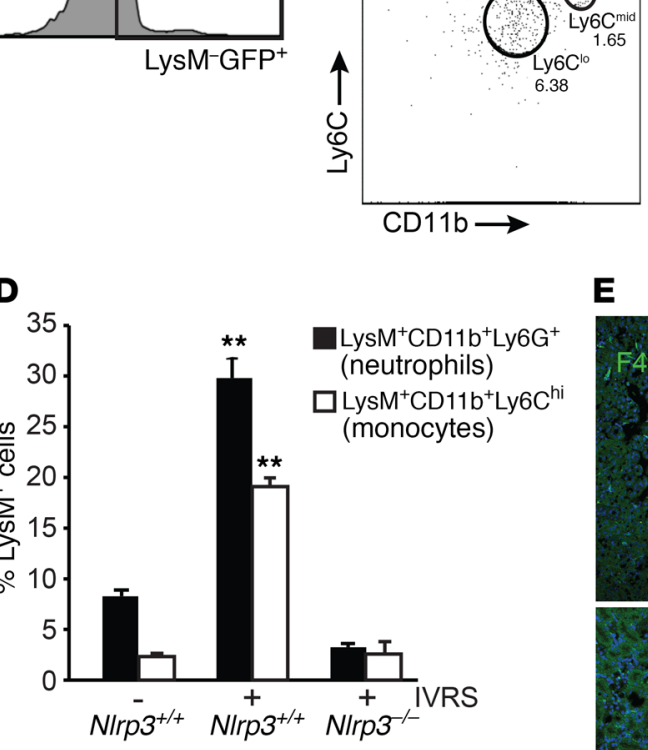

D

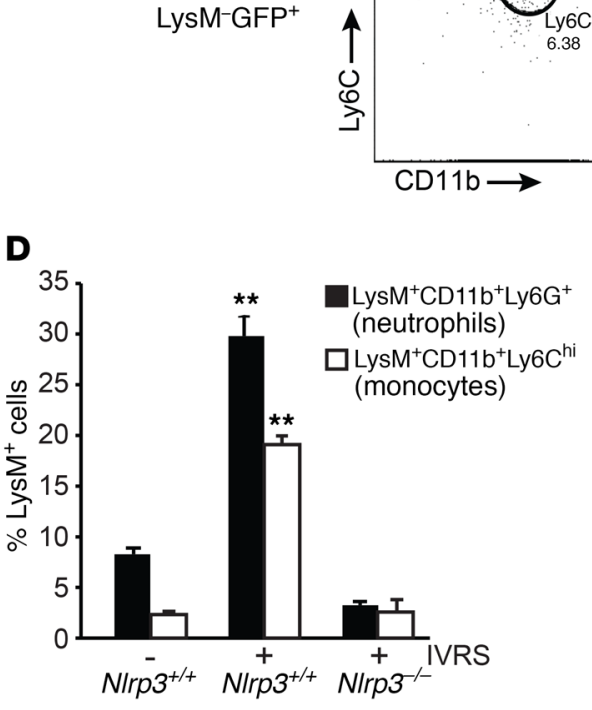

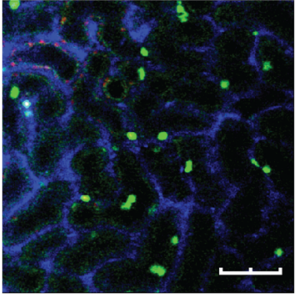

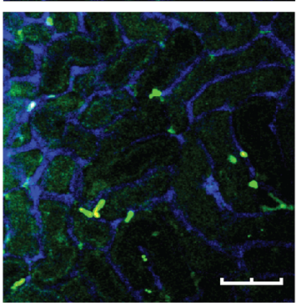

d 1
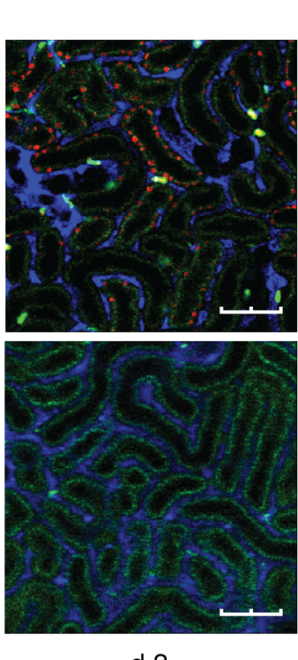

d 3
B

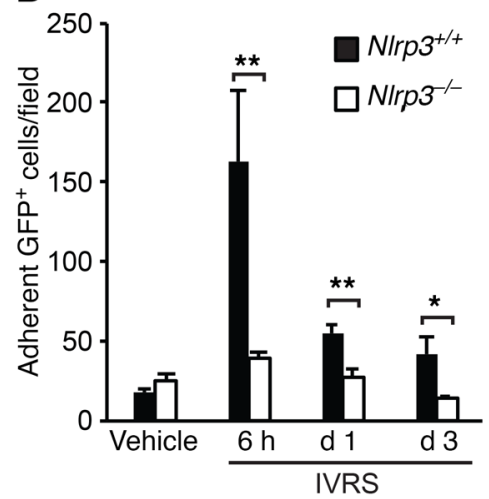

LysM-GFP

Tubules

Capillaries

SYTOX orange (injured cells)
E

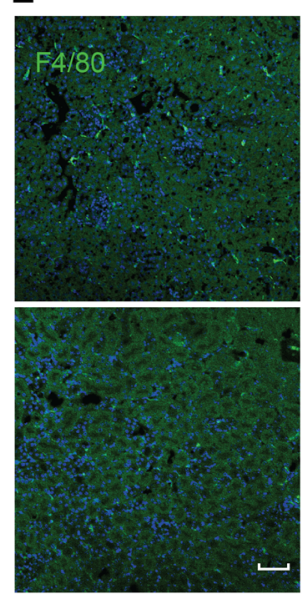

Vehicle

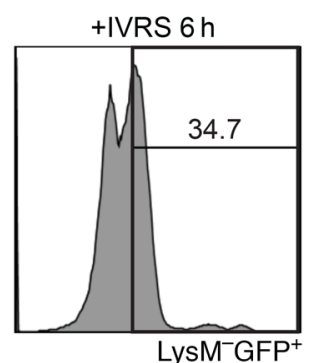

LysM-GFP $^{+}$
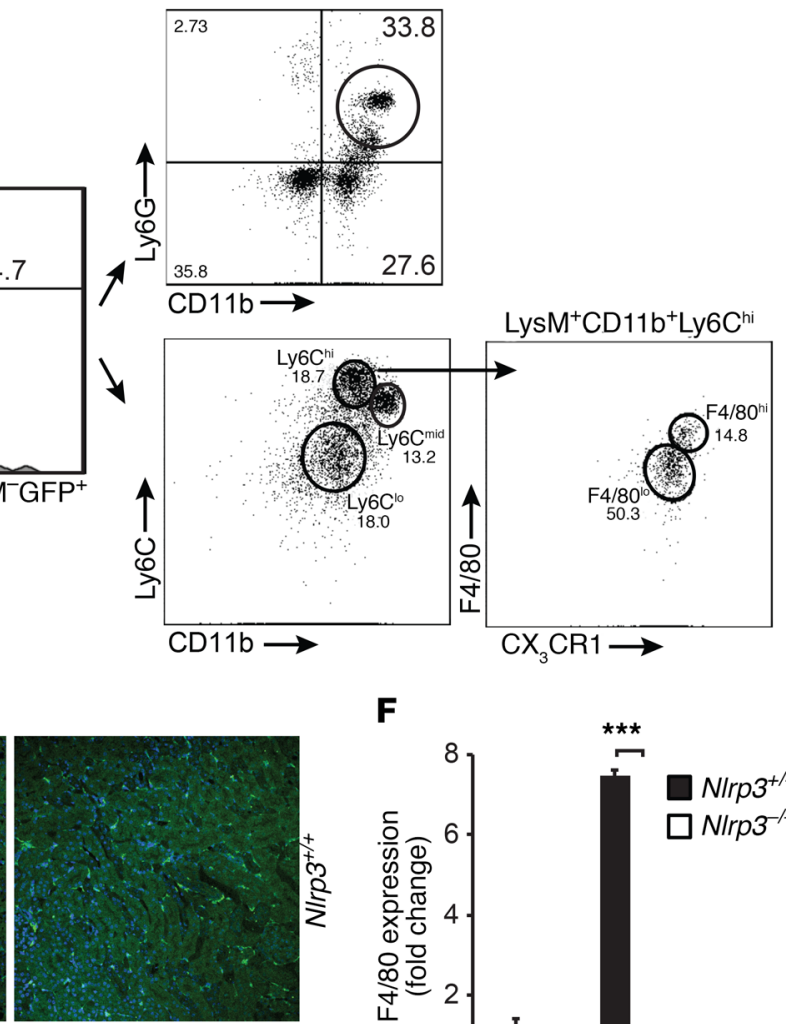

$\mathbf{F}$

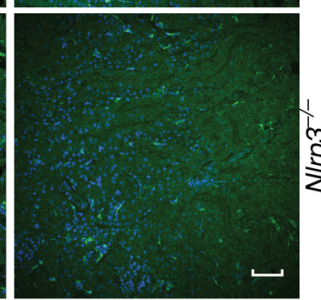

IVRS d 3

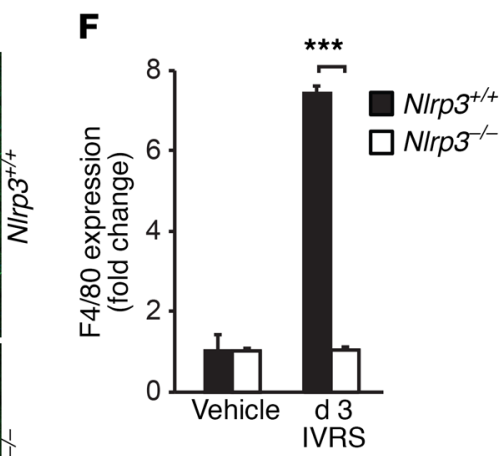


Figure 2. Contrast induces Nlrp3-dependent leukocyte recruitment to the kidney. (A) $L y s M^{(g f / / g f p)}$ and $N / r p 3^{-/-} L y S M^{(g f p / g f p)}$ mice were treated with vehicle or ioversol and assessed at 6 hours, 1 day, and 3 days using multiphoton intravital microscopy. Capillaries and injured/necrotic cells were labeled with Qtracker and SYTOX, respectively. Tubules are visualized by autofluorescence. Image is representative of 3 independent experiments. Scale bars: $100 \mu \mathrm{m}$. (B) Quantification of stationary GFP+ cells per field ( $\mathrm{NIrp3}^{+/+}$ vs. NIrp3 $3^{-1}, 6$ hours: ${ }^{*} P=0.003$, day $1:{ }^{* *} P=0.002$, day $3:{ }^{*} P=0.02$, $n=6-9 /$ group, ANOVA). (C and D) $L y s M^{(g f p / g f p)}$ mice were treated with vehicle or ioversol, and LysM-GFP+ leukocytes were sorted from total kidney tissue and analyzed by flow cytometry for CD11b, CX $C R 1, F 4 / 80$, Ly6G, and Ly6C at 6 hours. Renal infiltrating neutrophils $\left(\mathrm{NIrp3}^{+/+}\right.$, vehicle vs. ioversol, ${ }^{*} P=0.005, n=3$ /group, 2 -tailed Student's $t$ test) and monocytes $\left(\mathrm{Nlrp3}^{+/+}\right.$, vehicle vs. ioversol, ${ }^{* *} P=0.002, n=3 /$ group, 2 -tailed Student's $t$ test) were quantified in ioversol-treated $\mathrm{Nlrp3}^{+/+}$and $\mathrm{NIrp3}^{-/-}$ mice at 6 hours by flow cytometry. (E and F) F4/80 immunofluorescence (confocal microscopy) and quantification in kidneys from $\mathrm{Nlrp3}^{+/+}$and $\mathrm{Nlrp}^{-/-}$

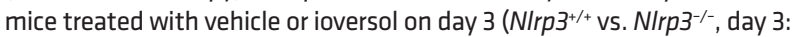
${ }^{* * *} P<0.001, n=3-5 /$ group, 2 -tailed Student's $t$ test). Scale bars: $200 \mu \mathrm{m}$.

cytes. Using multiphoton intravital microscopy, we demonstrate that contrast media is rapidly taken up by perivascular resident renal phagocytes to trigger leukocyte recruitment in an IL-1-dependent manner. Contrast activates the canonical Nlrp3 inflammasome in macrophages, which contributes to CI-AKI through the regulation of inflammation rather than via direct Nlrp3-mediated tubular cell death. Furthermore, we identify a mechanism of immune surveillance whereby monocytes/macrophages recruited to the kidney ingest contrast transported from the urine through direct interaction with tubular cells. The tubular reabsorption of contrast occurs via the brush border enzyme dipeptidase-1 (DPEP-1), another key event in the pathogenesis of CI-AKI. Together, these data increase the understanding of the interplay among resident renal phagocytes, tubular cells, and recruited leukocytes in kidney injury and provide opportunities to develop specific and effective therapies for CI-AKI in humans.

\section{Results}

Nlrp3 is required for CI-AKI. Our studies and others have demonstrated a role for Nlrp3 in renal inflammation and tubular cell injury in kidney disease models (12-15). To dissect the role of Nlrp3 in CI-AKI, we administered the non-ionic low-osmolar contrast agent ioversol to volume-depleted mice (Supplemental Figure 1; supplemental material available online with this article; https:// doi.org/10.1172/JCI96640DS1). Although volume depletion alone resulted in a rise in the serum creatinine level, in WT mice, ioversol induced AKI with a greater increase in serum creatinine at 72 hours (Figure 1A). Nlrp3 $3^{---}$mice were protected from the effects of contrast, maintaining stable kidney function. Kidney injury marker 1 (KIM-1), a marker of acute tubular injury, was also significantly increased in WT compared with $N \operatorname{lrp3^{-/}}$ mice (Figure 1, B and C). Tubular cell injury increased in $\mathrm{Nlrp3}^{+/+}$compared with $\mathrm{Nlrp}^{-/-}$ mice at 72 hours as determined by kidney intravital microscopy and labeling with the cell membrane impermeable dye SYTOX orange (Figure 1, D and E).

To better understand the interplay between different cellular compartments within the kidney during CI-AKI, we employed multiphoton intravital microscopy of the kidney in $L y s M^{(s f p / g f p)}$ and $N \operatorname{lrp3^{-/}} L y s M^{(g f p / g f p)}$ mice. Lys $M^{(g f p / g f p)}$ reporter mice express the flu- orescent GFP protein on cells of myeloid lineage, such as circulating monocytes/macrophages and granulocytes (17). The onset of detectable SYTOX orange-positive injured tubular cells occurred at approximately 24 hours after contrast administration and increased over 72 hours in $L y s M^{(g f p / g f p)}$ but not $N \operatorname{lrp} 3^{-/} L y s M^{(g f p / g f p)}$ mice (Figure 2A). Interestingly, visible tubular cell injury was preceded by a wave of $\mathrm{GFP}^{+}$leukocyte recruitment to the kidney at 6 hours that diminished over 24-72 hours (Figure 2, A and B, and Supplemental Videos 1-3). Crawling and adherent LysM-GFP ${ }^{+}$cells were observed in the peritubular capillaries adjacent to tubules in the tubulointerstitial space and within the tubules themselves. In $N l r p 3^{-/-} L y s M^{(f f p / g f p)}$ mice, the recruitment of $\mathrm{GFP}^{+}$leukocytes was largely absent following ioversol administration, with levels similar to those in vehicle-treated mice (Figure 2, A and B, and Supplemental Videos 1-3). To identify the cell populations infiltrating the kidney during CI-AKI, we used flow cytometry to sort and analyze $\mathrm{GFP}^{+}$cells isolated from $\operatorname{Lys} M^{(g f / g(s p)}$ kidney tissue homogenates at 6 hours. In untreated mice, a small number of $\mathrm{LysM}^{+} \mathrm{CD} 11 \mathrm{~b}^{+} \mathrm{Ly} 6 \mathrm{C}^{+}$ and $\mathrm{LysM}^{+} \mathrm{CD} 11 \mathrm{~b}^{+} \mathrm{Ly} 6 \mathrm{G}^{+}$leukocytes were isolated from the kidney, representing primarily circulating monocytes/macrophages, neutrophils, and possibly a few resident renal phagocytes (Figure 2, C and D). At 6 hours following contrast administration, an increase in kidney LysM-GFP ${ }^{+}$leukocytes was observed, with approximately $30 \%$ of the recruited cells representing $\mathrm{CD}_{11 \mathrm{~b}^{+} \mathrm{Ly} 6 \mathrm{G}^{+} \text {neutro- }}$ phils and $20 \% \mathrm{CD}_{11} \mathrm{~b}^{+} \mathrm{CX}_{3} \mathrm{CR} 1^{+} \mathrm{F} 4 / 80^{+} \mathrm{Ly}_{6 \mathrm{C}^{\text {hi }}}$ Ly6G ${ }^{-}$monocytes/ macrophages (Figure 2, C and D). Although the number of recruited $\mathrm{GFP}^{+}$leukocytes decreased by 24 hours in $L y s M^{(g / p / g f p)}$ mice, an increase in the number of $\mathrm{F} 4 / 80^{+}$macrophage population remained at 72 hours (Figure 2, $\mathrm{E}$ and $\mathrm{F}$ ). An increase in $\mathrm{F} 4 / 80^{+}$renal macrophages in the kidney was not observed in $\mathrm{Nlrp}^{-/-}$mice, consistent with the overall decrease in inflammation and AKI in this strain. Taken together, these data show that Nlrp3 is required for CI-AKI. Nlrp3 regulates neutrophil and monocyte/macrophage recruitment to the kidney in response to contrast administration that precedes the development of visible tubular epithelial cell (TEC) injury.

Contrast-induced epithelial cell toxicity does not require Nlrp3. Numerous studies have demonstrated the direct cytotoxic effect of contrast media on renal TECs $(1,18,19)$. Primary human proximal TECs (HPTCs) were treated with ioversol or the ionic contrast agent diatrizoate (DTA), and cell viability was examined. Not surprisingly, the two contrast agents were equally capable of inducing cell death in HPTCs within 24 hours as determined by MTT assay (Figure 3, A and B). Fluorescence live cell imaging with propidium iodide confirmed contrast-induced progressive necrotic cell death starting within 6 hours (Figure 3C and Supplemental Figure 2A). The cytotoxic effects of contrast agents diminished with dilution that would be expected to occur in most clinical scenarios, such as in patients receiving i.v. contrast. Importantly, there are significant confounding variables in the in vitro assay due to supraphysiologic osmotic load and the dilution of nutrients caused by the addition of contrast agents to cell culture media. Cell viability was also substantially reduced in control experiments using equiosmolar mannitol solutions or dilution of culture media with equivalent volumes of PBS (Supplemental Figure 2B). However, cell death above experimental conditions controlling for osmolarity or nutrient deficiency confirmed that ioversol exhibits inherent properties that are toxic to epithelial cells. 
A

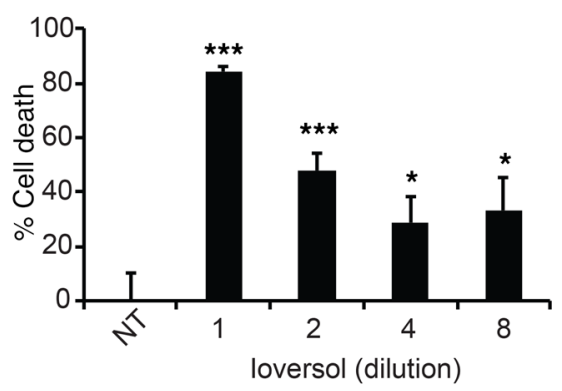

D

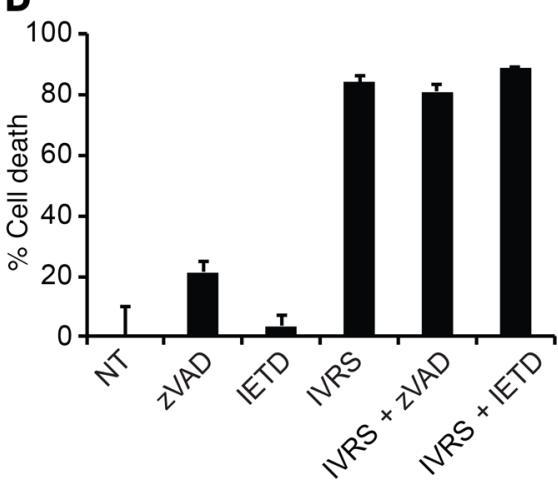

B

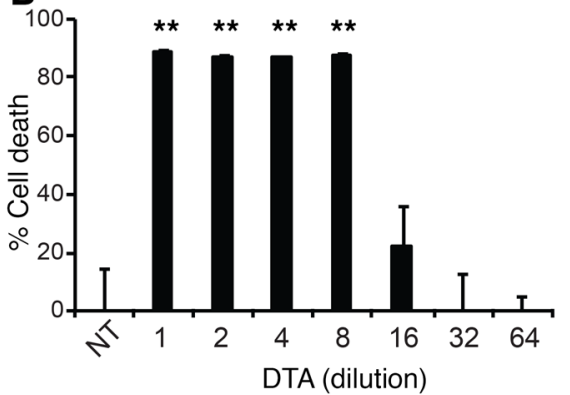

$\mathbf{E}$

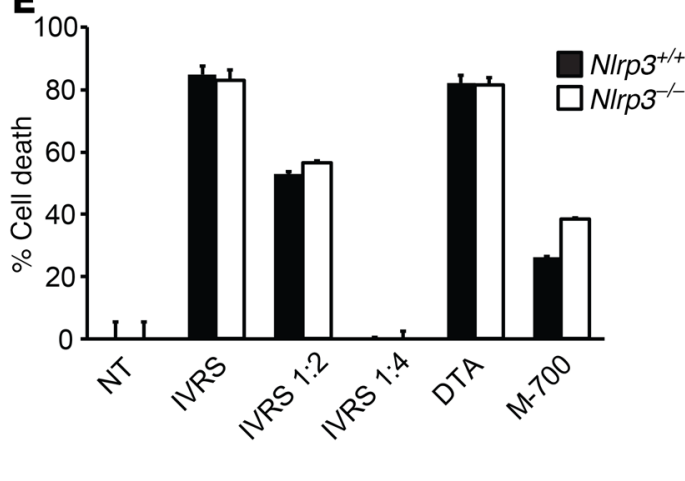

C

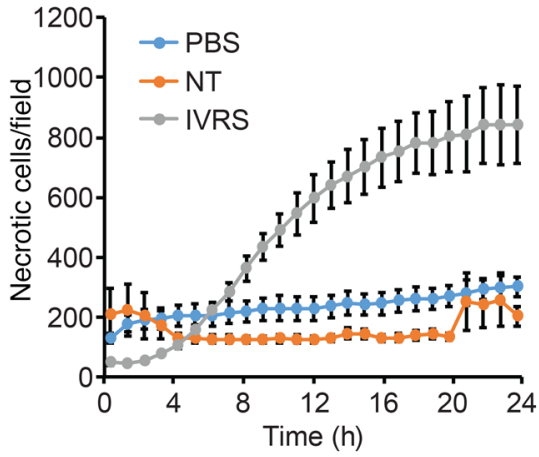

Figure 3. Contrast cytotoxicity in renal TECs. (A and B) Cell death was determined in HPTCs using MTT assay after treatment with various dilutions of ioversol in culture medium (1:1 to $1: 8)$ (vs. no treatment [NT]), 1:1: ${ }^{* *} P<0.001,1: 2:{ }^{* * *} P<0.001,1: 4:{ }^{*} P=0.03,1: 8:{ }^{*} P=0.03, n=9-12 /$ group, ANOVA) or DTA (1:1 to 1:64) (vs. NT, 1:1: ${ }^{* *} P=0.008,1: 2:{ }^{* *} P=0.009,1: 4:{ }^{*} P=0.009,1: 8:{ }^{*} P=0.009, n=9-12 /$ group vs. NT, ANOVA). (C) HPTCs were treated with PBS control or ioversol and labeled with propidium iodide to identify necrotic cells for 24 hours. Images were captured and analyzed for necrotic cells with the IncuCyte Live Cell Analysis System ( $n=4$ /group). (D) Cell death in ioversol-treated HPTCs with pan-caspase (zVAD) or caspase-8 (IETD) inhibitors (MTT assay, $P=$ NS, $n=6-12$ / group, ANOVA). (E) TECs isolated from Nlrp3 $3^{+/+}$or Nlrp3 $3^{-/-}$mice were treated with ioversol, DTA, or hyperosmolar mannitol buffer (700 mOsm) (M-700). Cell death was measured by MTT assay ( $P=\mathrm{NS}, n=4$ /group, ANOVA).

We previously demonstrated a noncanonical role for Nlrp3 in TEC apoptosis (11). To determine whether Nlrp3-mediated CI-AKI in vivo occurred via its effect on tubular epithelial death, we explored cell death pathways induced by contrast agents in HPTCs. Immunoblotting revealed a lack of apoptosis, as ioversol, regardless of dose, did not activate PARP cleavage, caspase- 8 activation, or caspase-3 activation (Supplemental Figure 3A). At high concentrations only, ioversol induced receptor-interacting protein 3 (RIP-3) and mixed lineage kinase domain-like (MLKL) phosphorylation in the necroptosis pathway, which has been linked to the Nlrp3 inflammasome (ref. 20 and Supplemental Figure 3B). However, RIP-1 (upstream of RIP-3) and MLKL inhibitors - necrostatin-1 and necrosulfonamide, respectively - only modestly attenuated contrast-induced cell death, and primary TECs isolated from Rip $3^{---}$mice were not protected (Supplemental Figure 3, C and D). Consistent with these findings, HPTCs were not protected from contrast by pan-caspase or caspase-8 inhibitor zVAD or IETD, respectively, and TECs isolated from $\mathrm{Nlrp3}^{-/-}$mice were as susceptible to contrast-induced cell death as WT cells (Figure 3, D and E). Contrast-induced HPTC death in vitro appeared to be mediated primarily by ferroptosis, with contrast inducing substantial lipid peroxidation that was blocked by ferrostatin-1 (Supplemental Figure 4). These results demonstrate that contrast-induced cytotoxicity in vitro is likely mediated by multiple pathways and potential confounding experimental factors. Furthermore, the pathogenesis of CI-AKI in vivo does not simply involve direct effects of Nlrp3 on epithelial cell death pathways.
Contrast activates the canonical Nlrp3 inflammasome in macrophages. The preceding studies suggested that the role of Nlrp3 in CI-AKI may require the canonical inflammasome as opposed to directing epithelial cell death. Ioversol induced caspase-1 activation and IL- $1 \beta$ cleavage in THP- 1 macrophages as detected by immunoblotting (Figure 4A). The ionic contrast agent DTA also induced IL-1 $\beta$ secretion in macrophages at 6 hours (Figure $4 \mathrm{~B}$ ). Confocal microscopy confirmed the formation of caspase-1positive ASC specks at 6 hours following ioversol stimulation, indicative of active inflammasomes (Figure $4 \mathrm{C}$ ). To confirm that inflammasome activation by contrast agents was mediated by Nlrp3, we deleted Nlrp3 expression in THP-1 macrophages using CRISPR-Cas 9 gene editing. The absence of Nlrp3 completely abrogated ioversol-induced IL- $1 \beta$ activation, an effect that was also observed in mouse $\mathrm{Nlrp3}^{-/-}$bone marrow-derived macrophages (BMDMs) (Figure 4, D and E). Consistent with these data, WT mice receiving ioversol expressed higher levels of renal IL-1 $\beta$ at 6 hours in vivo compared with $N l r p 3^{-/-}$animals (Figure $4 \mathrm{~F}$ ). Bone marrow chimera studies demonstrated that most of IL-1 $\beta$ production in vivo was dependent on hematopoietic cells (leukocytes), since IL-1 $\beta$ levels were significantly increased in $\mathrm{Nlrp}^{-/-}$mice receiving $\mathrm{Nlrp} 3^{+/+}$bone marrow, compared with $\mathrm{Nlrp3}^{+/+}$mice receiving Nlrp $3^{-/-}$cells (Figure $4 \mathrm{G}$ ). Consistent with this result, there was no evidence of ioversol-induced inflammasome activation (caspase- 1 cleavage or IL-1 $\beta$ production) in mouse primary kidney pericytes or human glomerular endothe- 
lial cells (Supplemental Figure 5), supporting the notion that the majority of inflammasome activity induced by contrast in the kidney originates from the leukocytes.

To explore further the mechanism of inflammasome activation by contrast agents, we tested the contribution of damage-associated molecular patterns (DAMPs) released from ioversol-injured epithelial cells, since canonical inflammasome activation by radiographic contrast in leukocytes in vivo could be induced either directly by contrast or indirectly in response to contrast-mediated tubular injury. Both ioversol and DAMPs released from ioversol-injured HPTCs alone were sufficient to induce IL-1 $\beta$ release from THP-1 macrophages (Figure $4 \mathrm{H}$ ). The combination of HPTC DAMPs and ioversol, however, was not additive or synergistic, as IL-1 $\beta$ secretion increased only marginally compared with either stimulation alone. Finally, canonical inflammasome activation by ioversol was not affected by the RIP-1 inhibitor necrostatin-1, which has been previously shown to attenuate CI-AKI in vivo (ref. 19 and Supplemental Figure 6). Taken together, these data show that contrast induces the canonical Nlrp3 inflammasome primarily in leukocytes in vitro and in vivo.

Resident renal phagocytes and recruited leukocytes provide vascular and urinary immune surveillance in the kidney. Inflammation plays a significant role in the pathogenesis of multiple kidney diseases $(7,15)$; however, the mechanism underlying the initiation and propagation of an inflammatory response in kidney injury remains incompletely understood. Recent studies have shown that perivascular resident renal phagocytes play a key role in the immune surveillance of immune complexes in the circulation $(9,10)$. To further probe the mechanism of Nlrp3 inflammasome activation and the subsequent immune response triggered by contrast in vivo, we labeled DTA on its free carboxyl group with CF568 (CF568-DTA) to visualize the temporal handling of contrast in the kidney using intravital microscopy (ioversol is non-ionic, and labeling with CF568 was therefore not possible). In prehydrated $L y s M^{(g f p / g f p)}$ mice, contrast rapidly entered the peritubular capillary network, emerging quickly into the tubular lumen, and cleared within 5 minutes (Figure 5A and Supplemental Video 4). The entry into the tubular lumen likely occurred via glomerular filtration, as no direct transit of contrast from the vascular space to the tubulointerstitial compartment was observed. Consequently, in prehydrated $L y s M^{(g f / g f p)}$ mice, no leukocyte recruitment was seen. Interestingly, in prehydrated $C X_{3} C R 1^{(g f p /+)}$ mice, significant uptake of CF568-DTA was detected in resident $\mathrm{CX}_{3} \mathrm{CR}^{+}$renal phagocytes within 30 seconds, confirming a potential mechanism to activate the Nlrp3 inflammasome in these cells (Figure 5B). Next CF568-DTA was administered to volume-depleted mice. As in prehydrated mice, CF568-DTA rapidly appeared in the peritubular capillary network, followed by entry into the tubular lumen likely by glomerular filtration (Figure 5, A and C, and Supplemental Video 5). Rapid contrast uptake into $\mathrm{CX}_{3} \mathrm{CR} 1^{+}$resident renal phagocytes was also observed by microscopy and confirmed by flow cytometry to include $\mathrm{CX}_{3} \mathrm{CR} 1^{+}$and $\mathrm{CD} 11 \mathrm{c}^{+}$cells (Figure 5, D and E, and Supplemental Video 6). Compared with the prehydrated state, CF568-DTA was reabsorbed, accumulated, and retained in tubules in a patchy manner over 40 minutes (Figure 5, A and C, and Supplemental Videos 5 and 7). The amount of contrast accumulating in the tubules, however, constituted a small fraction of the total administered contrast, since the majority was seen in the bladder of the mice at 1 hour by x-ray imaging (Supplemental Figure 7). In volume-depleted $L y s M^{(\text {(ff/gfp) }}$ mice, the recruitment of $\mathrm{GFP}^{+}$leukocytes coincided with contrast accumulation in renal tubules (Figure 5A, Figure 6A, and Supplemental Figure 8A). Intravital microscopy over 60 minutes revealed an increased number of crawling of $\mathrm{GFP}^{+}$ leukocytes in the interstitial space along contrast-laden tubules, as well as migration into the tubular epithelial layer and tubular lumen (Figure 6B and Supplemental Video 8). Direct interactions between $\mathrm{GFP}^{+}$leukocytes and tubular cells were observed, with $\mathrm{GFP}^{+}$leukocytes actively taking up contrast material, detaching from the tubule, and migrating away from the field (Figure 6B and Supplemental Video 8). Unlike LysM-GFP ${ }^{+}$leukocyte migration and movement in the kidneys around contrast-laden tubules, $\mathrm{CX}_{3} \mathrm{CR} 1^{+}$dendritic cells showed little to no movement or change in position, extension, or retraction activity (Supplemental Figure 8B). Furthermore, similar uptake of filtered contrast from tubules to $\mathrm{CX}_{3} \mathrm{CR}^{+}$resident renal phagocytes was not observed (Supplemental Video 6), suggesting that this property was exclusive to the recruited leukocyte population. $\mathrm{CD}^{+} 5^{+} \mathrm{CF} 568-\mathrm{DTA}^{+}$leukocytes isolated from WT kidneys were analyzed by flow cytometry and found to be primarily Ly $6 \mathrm{C}^{+} \mathrm{F} 4 / 80^{+}$ but largely Ly $6 \mathrm{G}^{\text {neg/lo }}$, consistent with contrast uptake by recruited monocytes/macrophages (Figure 6C and Figure 7). Only a small population of $\mathrm{CD} 45^{+} \mathrm{CD} 11 \mathrm{~b}^{+} \mathrm{Ly} 6 \mathrm{G}^{\mathrm{hi}}$ neutrophils was present in the kidney 1 hour after contrast administration, and very few of these cells were CF568-DTA ${ }^{+}$(Figure 6C). Control experiments using CF568 alone or a mock preparation of CF658-acetate in volumedepleted mice demonstrated no tubular accumulation or inflammatory response, confirming that the effects seen were dependent on the DTA contrast agent (Supplemental Figure 9). Contrast uptake by recruited monocytes/macrophages was dependent on ICAM-1, a leukocyte adhesion molecule. In mice pretreated with blocking anti-ICAM-1 antibody, a reduction in recruited $\mathrm{CD}_{11 \mathrm{~b}} \mathrm{CX}_{3} \mathrm{CR} 1^{+}$ Ly $6 \mathrm{C}^{+} \mathrm{F} 4 / 80^{+}$cells was observed in response to CF568-DTA at 1 hour. Furthermore, in the remaining kidney monocytes/macrophages, the proportion of CF568-DTA ${ }^{+}$cells was reduced compared with that in IgG control-treated mice (Figure 7). Uptake of contrast by monocytes/macrophages was not dependent on scavenger receptor A (SR-A), since the apolipoprotein-A1 mimetic and SR-A binding peptide $\mathrm{D}-4 \mathrm{~F}(21,22)$ had minimal effect on the proportion of recruited monocytes/macrophages taking up DTA (Figure 7). Of note, leukocyte uptake of CF568-DTA was not detectable in mice at 6 hours, likely due to clearance of the fluorescent contrast reagent from the kidney (Supplemental Figure 10). Thus, during CI-AKI, early immune surveillance occurs from both resident renal phagocytes and recruited monocytes/macrophages. Resident renal phagocytes sample contrast from the circulation, while recruited monocytes/ macrophages take up contrast transported from the urine through direct interaction with tubular cells.

Contrast activates the inflammasome in resident renal phagocytes and infiltrating monocytes/macrophages. The previous experiments demonstrated that radiographic contrast activates the inflammasome in macrophages in vitro and that resident and infiltrating macrophages take up contrast in vivo. Flow cytometry was used to demonstrate inflammasome activation in these cell populations in vivo (Figure 8). At 6 hours following ioversol administration to

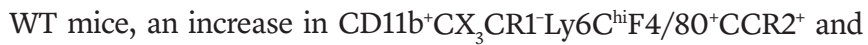
$\mathrm{CD}_{11} \mathrm{~b}^{+} \mathrm{CX}_{3} \mathrm{CR}^{+}{ }^{+} \mathrm{Ly} 6 \mathrm{C}^{\mathrm{hi}} \mathrm{F} 4 / 8 \mathrm{O}^{+} \mathrm{CCR} 2^{+}$monocytes/macrophages was 
A

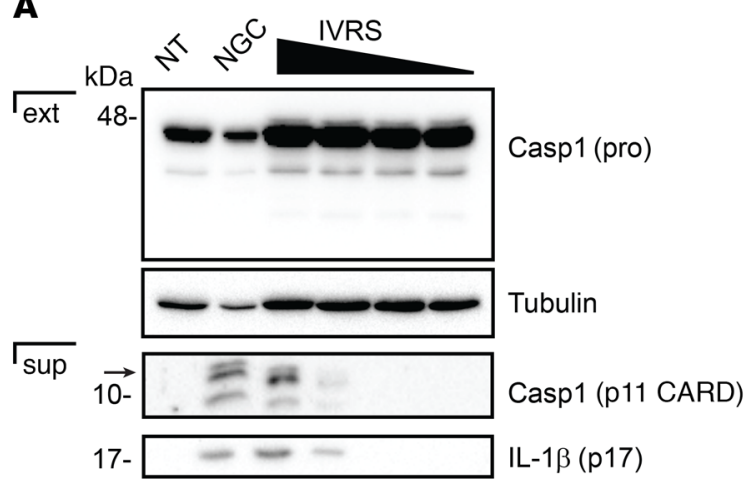

B

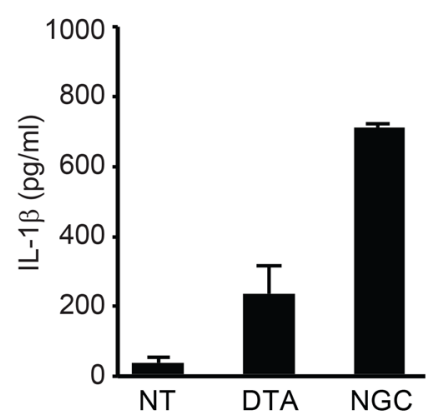

C
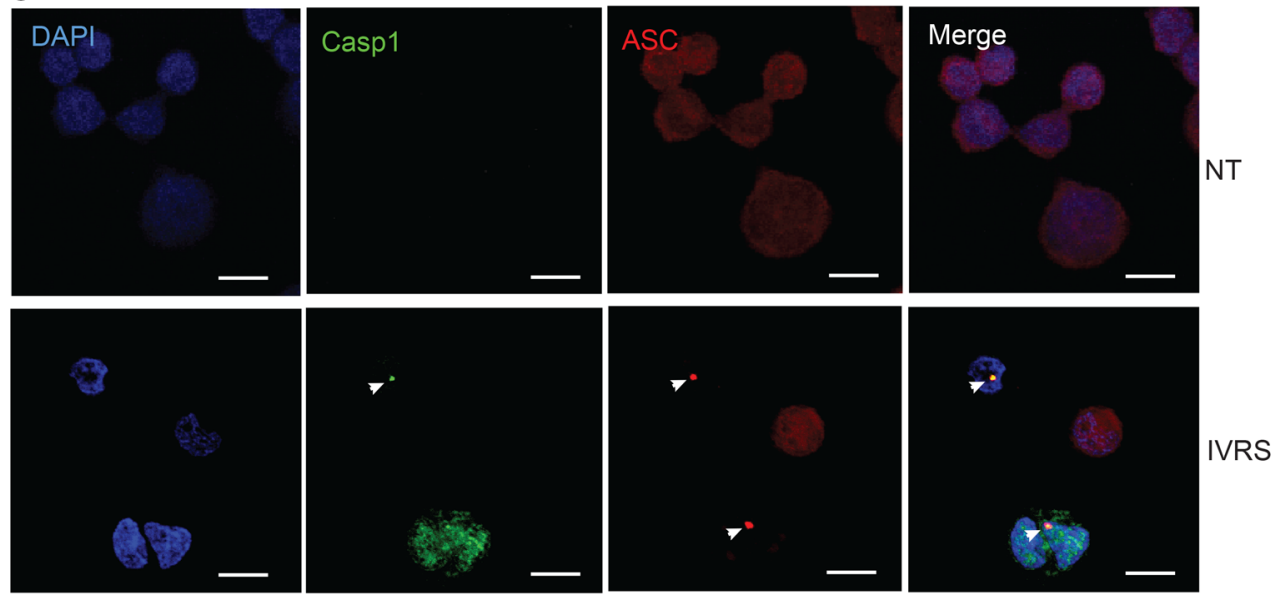

D

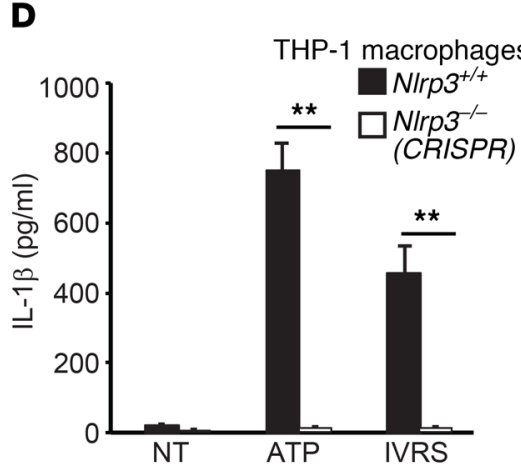

G

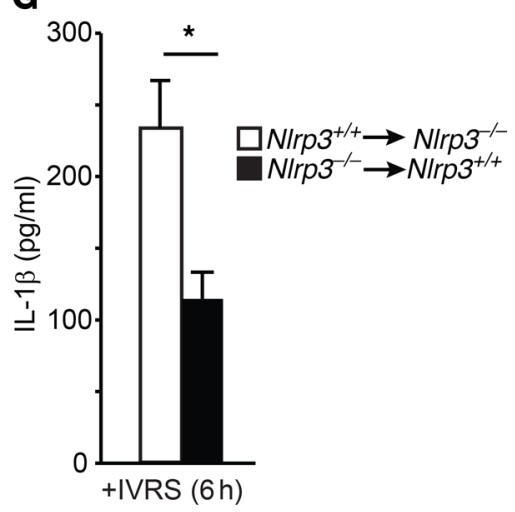

\begin{abstract}
E
\end{abstract}

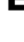

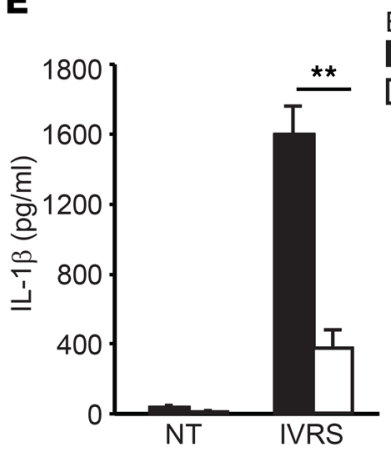

H

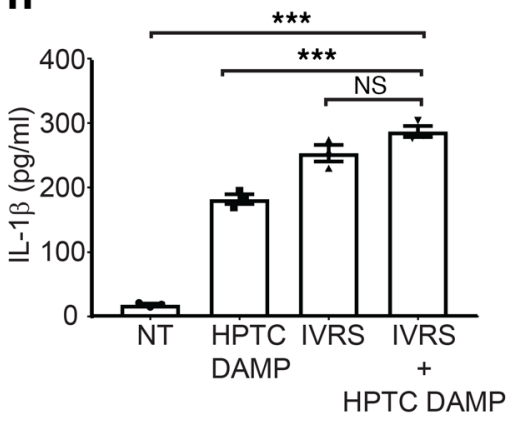

\section{$\mathbf{F}$}

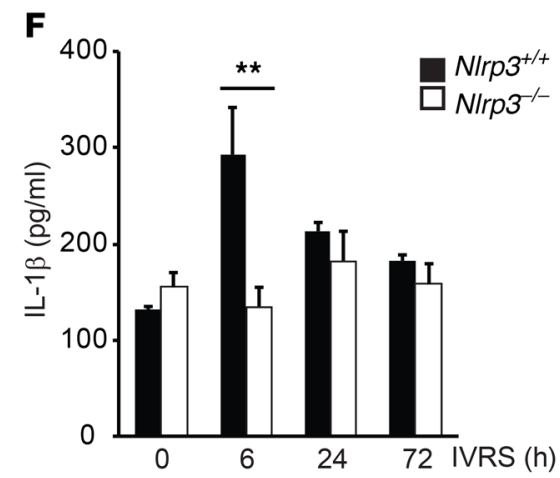


Figure 4. Contrast activates the canonical NIrp3 inflammasome in macrophages. (A) Immunoblot probing for caspase-1 (Casp1) (pro- and cleaved, p11 CARD domain) and cleaved IL-1 $\beta$ (p17) in extracts (ext) and supernatants (sup) of THP-1 cells untreated (NT) or treated with ioversol (1:1 to 1:8 dilution) at 6 hours. Nigericin (NGC) was used as positive control. Image is representative of 3 independent experiments. (B) THP-1 cells were treated with DTA or nigericin (NGC). IL-1 $\beta$ production was measured using ELISA at 6 hours ( $n=3$ /group). (C) Confocal microscopy for ASC or activated caspase- 1 in ioversol-treated THP-1 cells at 6 hours. Arrowheads indicate ASC- and caspase-1-positive specks. Image is representative of 3 independent experiments. Scale bars: $10 \mu \mathrm{m}$. (D) IL-1 $\beta$ ELISA in $\mathrm{Nlrp3}^{+/+}$and CRISPR-deleted NIrp3 $3^{-/-}$THP-1 cells treated with ioversol or the positive control ATP (NIrp3 ${ }^{+/+}$vs. Nlrp3 ${ }^{-1-}$, ATP: ${ }^{* *} P=0.01$, ioversol: ${ }^{* *} P=0.01$, $n=3$ /group, ANOVA). (E) IL-1 $\beta$ ELISA from Nlrp3 $3^{+/}$and $\mathrm{Nlrp3}^{-/-}$mouse BMDMs treated with ioversol at 6 hours ( $\mathrm{Nlrp3}^{+/+}$vs. NIrp3 $3^{-/-}$, IVRS: ${ }^{*} P=0.01, n=3 /$ group, 2 -tailed Student's $t$ test). (F) IL-1 $\beta$ ELISA in kidneys of $\mathrm{Nlrp3}^{+/+}$and $\mathrm{Nlrp3}^{-/-}$mice treated with ioversol over 3 days $\left(\mathrm{Nlrp3}^{+/+}\right.$vs. Nlrp3 $3^{-/-}, 6$ hours: ${ }^{* *} P=0.006, n=3-5 /$ group, ANOVA). (C) $\mathrm{NIrp3}^{+/+}$and $\mathrm{NIrp3}^{-/-}$mice were depleted using irradiation and clodronate-liposomes before bone marrow (BM) transplant ( $\mathrm{Nlrp3}^{+/+} \mathrm{BM}$ to $\mathrm{Nlrp3^{-/- }}$, N/rp3 $3^{-/-} \mathrm{BM}$ to

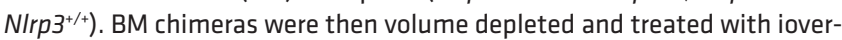
sol for 6 hours, and IL-1 $\beta$ ELISA was performed from kidney lysates ( ${ }^{*} P=0.03, n=4$ /group, 2-tailed Student's $t$ test). (H) DAMPs were generated from HPTCs using ioversol and used with or without ioversol to activate the inflammasome in THP-1 cells as measured by IL-1 $\beta$ ELISA. NT vs. ioversol+HPTC DAMPs, ${ }^{* * *} P=0.0001, n=3 /$ group; HPTC DAMPs vs. ioversol+HPTC DAMPs, ${ }^{* * *} P=0.0008, n=3 /$ group, ANOVA.

observed in the kidney, likely recruited from the circulation given the very high expression of Ly6C. In contrast, $\mathrm{CD} 11 \mathrm{~b}^{+} \mathrm{CX}_{3} \mathrm{CR} 1^{+}$

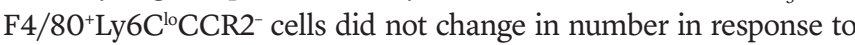
contrast and likely represented the resident phagocyte population (Figure 8A). In all of these monocyte/macrophage populations, we detected active caspase-1 using a caspase-1-FLICA probe at 6 hours but not 1 hour after ioversol administration, similar to the in vitro kinetics (Figure 8A and Supplemental Figure 11A). Consistent with these data, isolated $\mathrm{CX}_{3} \mathrm{CR}^{+}$resident renal phagocytes produced IL-1 $\beta$ in vitro when stimulated with ioversol or the canonical Nlrp3 agonist ATP, confirming the ability of these cells to activate the inflammasome in response to contrast and other DAMPs (Supplemental Figure 11B). On the contrary, $\mathrm{LysM}^{+} \mathrm{CD} 11 \mathrm{~b}^{+} \mathrm{Ly}_{6 \mathrm{G}}{ }^{+}$neutrophils did not demonstrate any evidence of caspase- 1 activation in vivo at 1 hour or 6 hours after ioversol administration (Supplemental Figure 12). Consistent with the induction of the proinflammatory cytokine IL-1 $\beta$ in vivo, $\mathrm{LysM}^{+} \mathrm{CD} 11 \mathrm{~b}^{+} \mathrm{CX}_{3} \mathrm{CR} 1^{+} \mathrm{F} 4 / 80^{+} \mathrm{Ly}_{6 \mathrm{C}} \mathrm{C}^{+}$monocytes/ macrophages acquired a proinflammatory phenotype over 72 hours following contrast administration with increasing CCR2 expression, an effect that was not observed in $\mathrm{Nlrp}^{3-/-}$ mice (Figure 8B). Together, these data show that contrast activates the inflammasome in resident and infiltrating monocytes/macrophages to drive a proinflammatory phenotype during CI-AKI.

Resident renal phagocytes and recruited leukocytes are required for CI-AKI. To confirm that inflammation regulated by resident renal phagocytes and other infiltrating leukocytes was an essential component of CI-AKI, we performed cell depletion and cytokine inhibition studies. Anti-Ly6G (clone 1A8) was used to deplete circulating neutrophils as well as some monocyte/macrophage populations in $L y s M^{(g f p / g f p)}$ mice prior to ioversol administration. In anti-Ly6G-compared with IgG control-treated mice, a significant reduction in adherent $\mathrm{GFP}^{+}$leukocytes was observed in the kidney at 6 hours (Figure 9, A and B). Consistent with this finding, renal function and serum creatinine were improved in Ly6G-depleted but not control IgG-treated mice (Figure 9B). Similar results were obtained when leukocyte recruitment was inhibited using blocking ICAM-1 antibodies (Figure 7 and Supplemental Figure 13A). Next, experiments were performed using clodronate-liposomes, which deplete resident renal phagocytes and some systemic macrophage populations (7). Clodronate-liposomes were administered to $L y s M^{(g f / g f p)}$ mice, and the population of resident renal phagocytes was confirmed depleted by F4/80 staining and flow cytometry (Figure 9C and Supplemental Figure 13B). In clodronateliposome- compared with control liposome-treated mice, macrophage depletion was associated with reduced $\mathrm{GFP}^{+}$leukocyte recruitment at 6 hours and improved kidney function at 3 days (Figure 9, C and D). These results were consistent with findings in diphtheria toxin-treated (DT-treated) CD11c-DTR transgenic mice (23). Since more than $90 \%$ of resident renal phagocytes express CD11c (8), DT treatment in this strain eliminates the majority of resident cells. Like clodronate-liposome-treated mice, CD11c-depleted animals displayed less early inflammation, confirming that resident renal phagocytes are necessary for leukocyte recruitment during CI-AKI (Supplemental Figure 13, C and D). Finally, to determine whether IL- $1 \beta$ played a significant role in mediating leukocyte recruitment during contrast administration, we pretreated $L y s M^{(g f / g f(p)}$ mice with the soluble IL-1 receptor antagonist IL-1Ra prior to contrast administration. Consistent with the prior data, IL-1Ra diminished $\mathrm{GFP}^{+}$leukocyte recruitment to the kidney at 6 hours, which translated into better renal function and serum creatinine at 3 days (Figure 9, E and F). KIM-1 expression and $\mathrm{F} 4 / 80^{+}$macrophage infiltration in the kidney was also reduced in IL-1Ra-treated mice at 3 days (Supplemental Figure 14). In summary, the resident renal phagocytes ICAM-1 and IL-1 regulate contrast-induced leukocyte recruitment to the kidney that is necessary for CI-AKI.

Renal DPEP-1 mediates tubular uptake of contrast in the urine. DPEP-1 is a glycosyl phosphatidylinositol-anchored (GPI-anchored) renal proximal tubule brush border enzyme that regulates the tubular uptake of imipenem as well as other tubular toxins, such as cisplatin, vancomycin, cyclosporine, and aminoglycosides (24-26). These studies suggest that DPEP-1 may function as a luminal receptor for a variety of molecules or drugs entering the urine following glomerular filtration. To determine whether DPEP-1 also regulates uptake of contrast into cells, we performed in vitro studies in primary HPTCs and DPEP-1-negative COS-1 cells transfected with human DPEP-1 (Figure 10, A and B, and Supplemental Figure 15, B and C). HPTCs expressed abundant DPEP-1 that was effectively deglycosylated using the endoglycosidase peptide:N-glycosidase F (PNGaseF) to impair DPEP-1 apical targeting and activity (ref. 27, Figure 10A, and Supplemental Figure 15A). HPTCs and DPEP-1-transfected COS cells internalized CF568-labeled DTA as detected by fluorescence live cell imaging, effects that were absent in PNGaseF-treated HPTCs and nontransfected COS cells (Figure 10, A and B, and Supplemental Figure 15, B-D). To test whether DPEP-1 mediated tubular uptake of contrast in vivo, we pretreated volume-depleted WT mice with the DPEP-1-specific inhibitor cilastatin prior to the administration of CF568-DTA and monitored them by intravital microscopy. Compared with control, cilastatin prevented tubular 
A

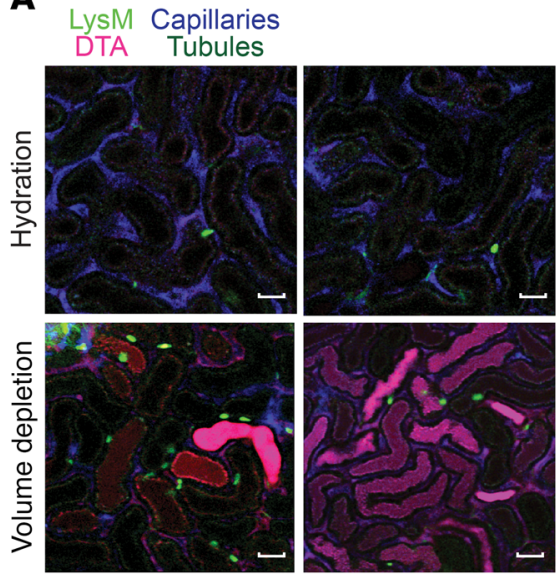

DTA568 (40 min)

C

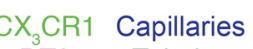

DTA Tubules

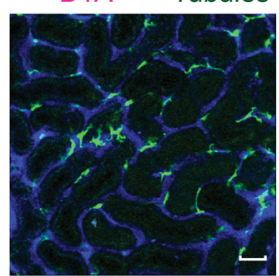

Before

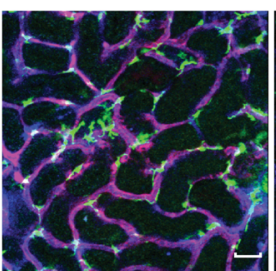

$1 \mathrm{~min}$

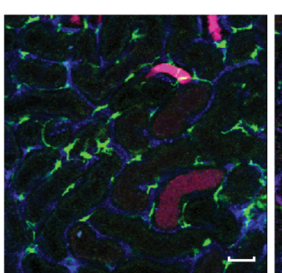

$20 \mathrm{~min}$
B ${ }_{C X} C R 1$

DTA
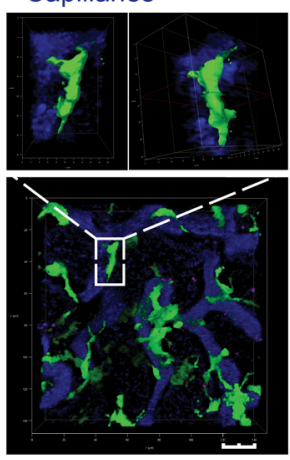

0 min
Capillaries
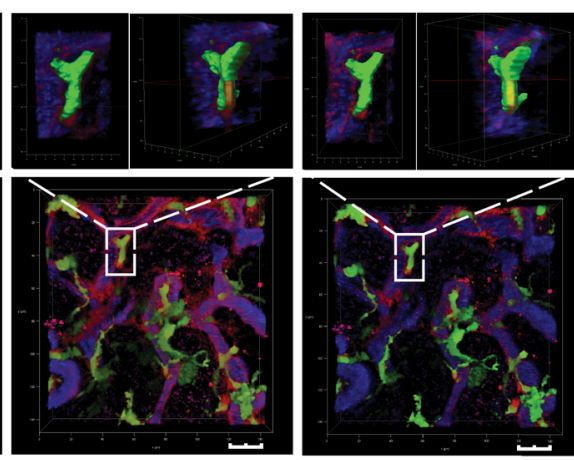

$30 \mathrm{~s}$

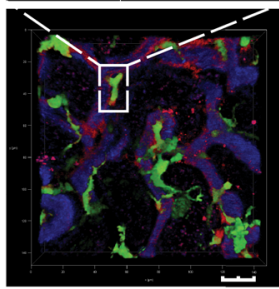

5 min

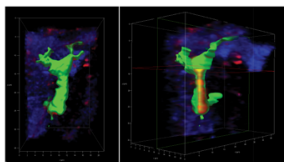

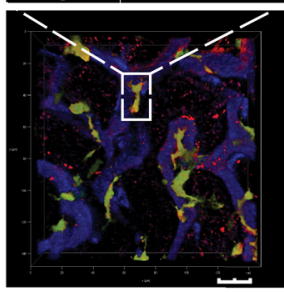

$10 \mathrm{~min}$
DTA568

D

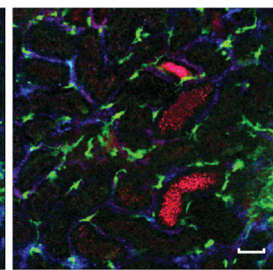

$40 \mathrm{~min}$

DTA568

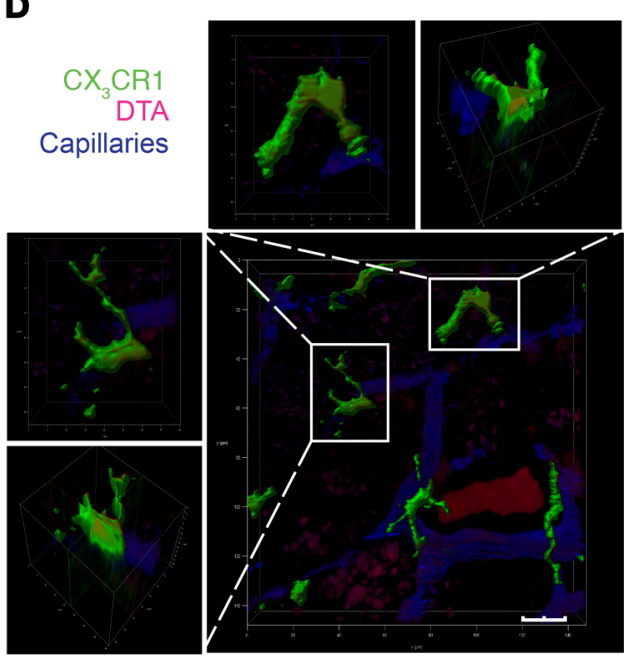

DTA568 (40 min)

E
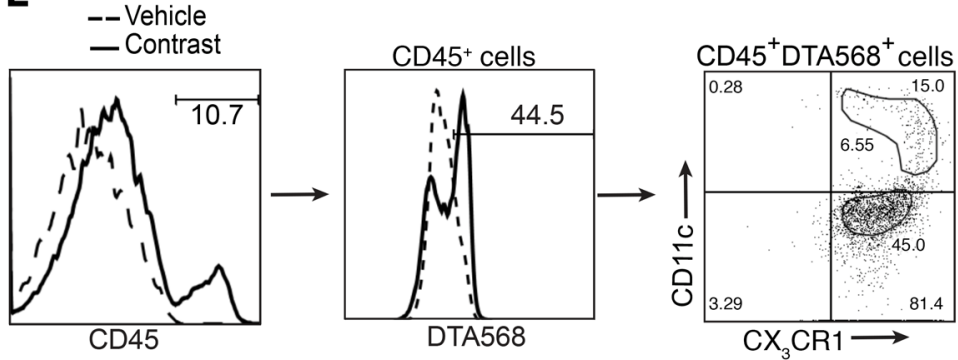

Figure 5. Contrast uptake and reabsorption in the kidney. (A) $L y s M^{(\lg f / g f p)}$ mice were injected with CF568- labeled DTA (DTA568) and imaged with multiphoton intravital microscopy. Upper panels: hydrated mice (i.v. normal saline); lower panels: volume-depleted mice. Capillaries were labeled with Qtracker, and tubules are visualized by autofluorescence. Images are representative of 3 independent experiments. Scale bars: $100 \mu \mathrm{m}$. (B) $\mathrm{Hydrated} C X_{3} C R 1^{(g f p /+)}$ mice were treated with CF568-labeled DTA and imaged with multiphoton intravital microscopy. 3D reconstruction (Z-stack) of DTA uptake in CX ${ }_{3}$ CR $1^{+}$resident phagocytes over 10 minutes. Images are representative of 3 independent experiments. Scale bars: $20 \mu \mathrm{m}$. (C) Tubular uptake of DTA in volume-depleted $C X_{3} C R 7^{(g f p /+)}$ mice over 40 minutes by multiphoton intravital microscopy. Images are representative of 3 independent experiments. Scale bars: $100 \mu \mathrm{m}$. (D) $3 D$ reconstruction (Z-stack) of DTA uptake in $C X_{3} C R 1^{+}$resident phagocytes in volume-depleted mice at 40 minutes. Images are representative of 3 independent experiments. Scale bar: $20 \mu \mathrm{m}$. (E) Mice were volume depleted and treated with CF568-labeled DTA for 1 hour, and contrast uptake was analyzed by flow cytometry in resident phagocytes ( $C D 45^{+} C X_{3} C R 1^{+} D T A^{+}$and $\left.C D 45^{+} C X_{3} C R 1^{+} C D 11 c^{+} D T A^{+}\right)$. Images are representative of 3 independent experiments.

uptake and retention of contrast in the kidney over 40 minutes (Figure 10C and Supplemental Video 9). In $L y s M^{(\text {(sp } /(f f)}$ mice, cilastatin effectively inhibited leukocyte recruitment at 6 hours and prevented oliguric renal injury at 1 and 3 days in response to ioversol, confirming also that the role of DPEP-1 in contrast handling and CI-AKI was not restricted to DTA (Figure 10, D-G). These data show that tubular reabsorption of contrast is mediated by DPEP- 1 that is also necessary for CI-AKI.

Inflammasome-related biomarkers are detected in humans undergoing coronary angiography. The previous results demon- strated early activation of the Nlrp3 inflammasome and inflammation in a mouse model of CI-AKI. To determine whether inflammasome activation could also be detected in humans receiving arterial contrast, we evaluated urine biomarkers in patients drawn from a prospective study of CI-AKI following coronary angiography. Forty-two stable patients undergoing elective coronary angiography were selected randomly from this cohort and their urine samples analyzed for inflammation biomarkers using the Meso Scale Discovery assay. Although patients in this study are being followed for AKI and CKD long-term, 


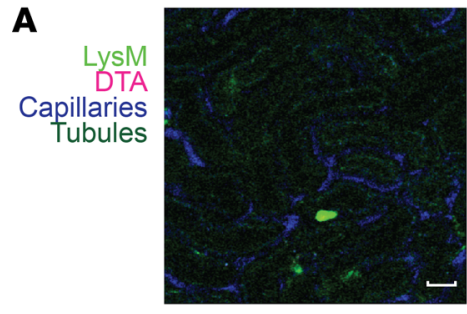

DTA568 Before

B

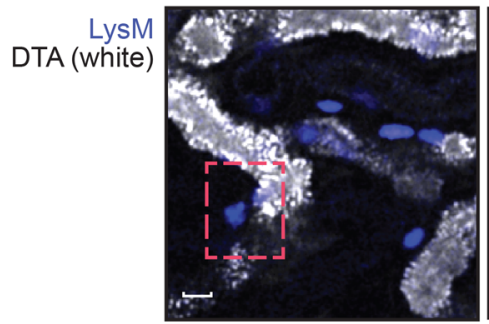

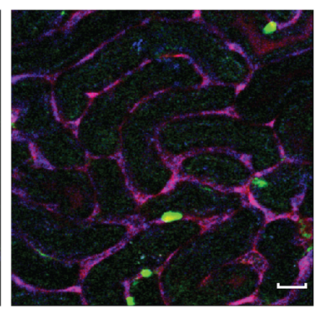

$1 \mathrm{~min}$

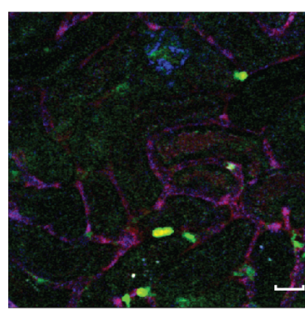

$20 \min$

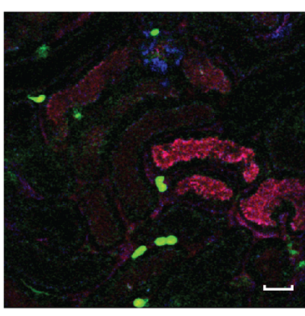

$40 \min$

DTA568 (40 $\mathrm{min})$
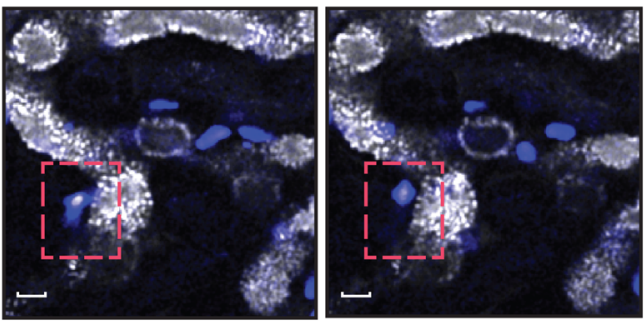

C
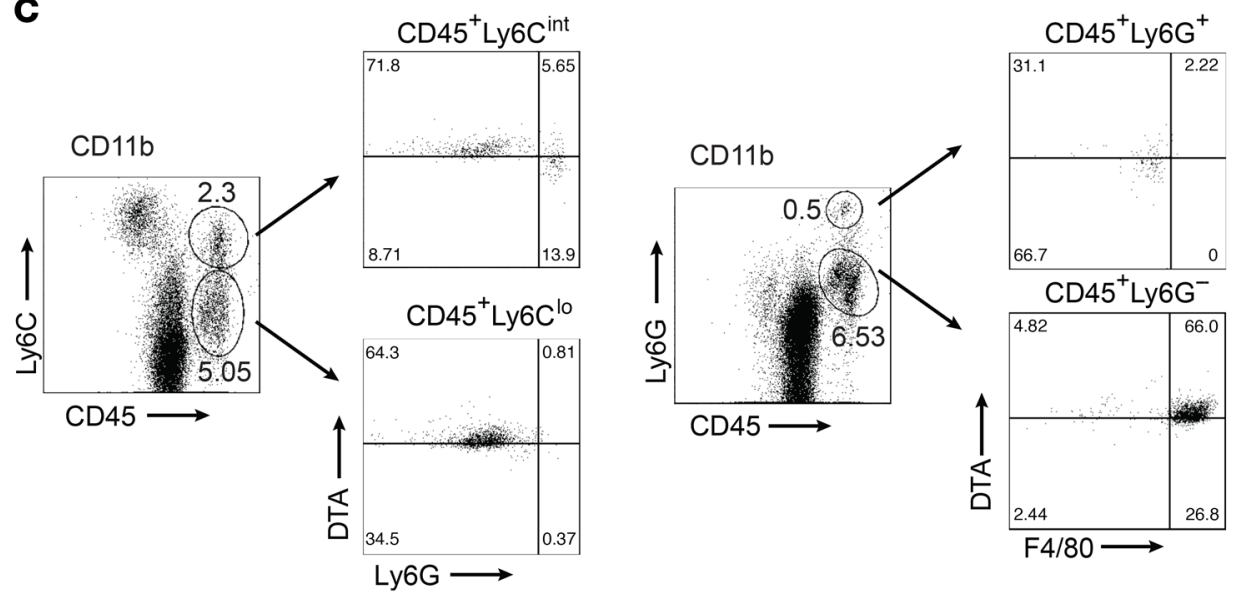

DTA568 $1 \mathrm{~h}$

Figure 6. Contrast uptake by infiltrating LysM-GFP+ leukocytes. (A) Lys $M^{(g f / g f f)}$ mice were injected with CF568-labeled DTA and imaged with multiphoton intravital microscopy. Capillaries were labeled with Qtracker, and tubules are visualized by autofluorescence. Images are representative of 3 independent experiments. Scale bars: $50 \mu \mathrm{m}$. (B) Infiltrating LysM-GFP+ leukocytes (blue) interacting directly with DTA-laden tubules (white) at 40 minutes (dashed red boxes). Images are representative of 3 independent experiments. Scale bars: $25 \mu \mathrm{m}$. (C) Flow cytometry characterization of contrast uptake in leukocytes isolated from mouse kidneys 1 hour after treatment with CF568-labeled DTA. Leukocytes were sorted using CD11b+, and CD45+, Ly6C+, Ly6C+', F4/80+ populations were analyzed for DTA uptake. Images are representative of 3 independent experiments.

urine samples were collected and analyzed before and immediately after angiogram ( $<24$ hours), before the onset of clinical AKI. All patients met the inclusion criteria of an estimated glomerular filtration rate (GFR) of less than $60 \mathrm{ml} / \mathrm{min} / 1.73$ $\mathrm{m}^{2}$ (CKD Epidemiology Collaboration [CKD-EPI] equation); patient characteristics are presented in Supplemental Table 1. The administration of arterial contrast significantly increased urinary expression of the inflammasome-dependent cytokine IL-18 as well as the tubular injury marker KIM-1 (Figure 11, A and B). In 4 of 8 randomly chosen patients from this cohort, cleaved caspase- 1 could be detected in the urine pellets after contrast administration (Figure 11C). To assess whether urinary caspase-1 was associated with the presence of leukocytes in the urine, we selected an additional 9 patients based the presence or absence of leukocyturia by urinalysis. In 5 patients, detectable urinary caspase-1 correlated with an increase in urine leukocytes after angiogram, whereas 4 patients with no leukocyturia did not show evidence of urinary caspase-1 (Figure 11D). In general, the presence of urinary caspase- 1 was associated with positive urine leukocytes by urinalysis (Figure 11E), consistent with the mouse data and the hypothesis that inflammasome activation by contrast occurs primarily in leukocytes. IL-1 $\beta$ was detectable at very low concentrations in the patient urine samples; however, a significant increase after contrast administration could not be detected. Similarly, multiple other biomarkers including IL-6, lipocalin-2 (NGAL), MCP-1, EGF, and IL-1 $\alpha$, were not significantly increased in patient urine immediately after contrast administration (data not shown). Finally, none of the biomarkers were predictive of AKI within 7 days. In a preliminary analysis, the incidence of CI-AKI in this stable patient cohort was low and less than $10 \%$, consistent with the multiple factors, aside from inflammation, needed to develop this clinical complication. 


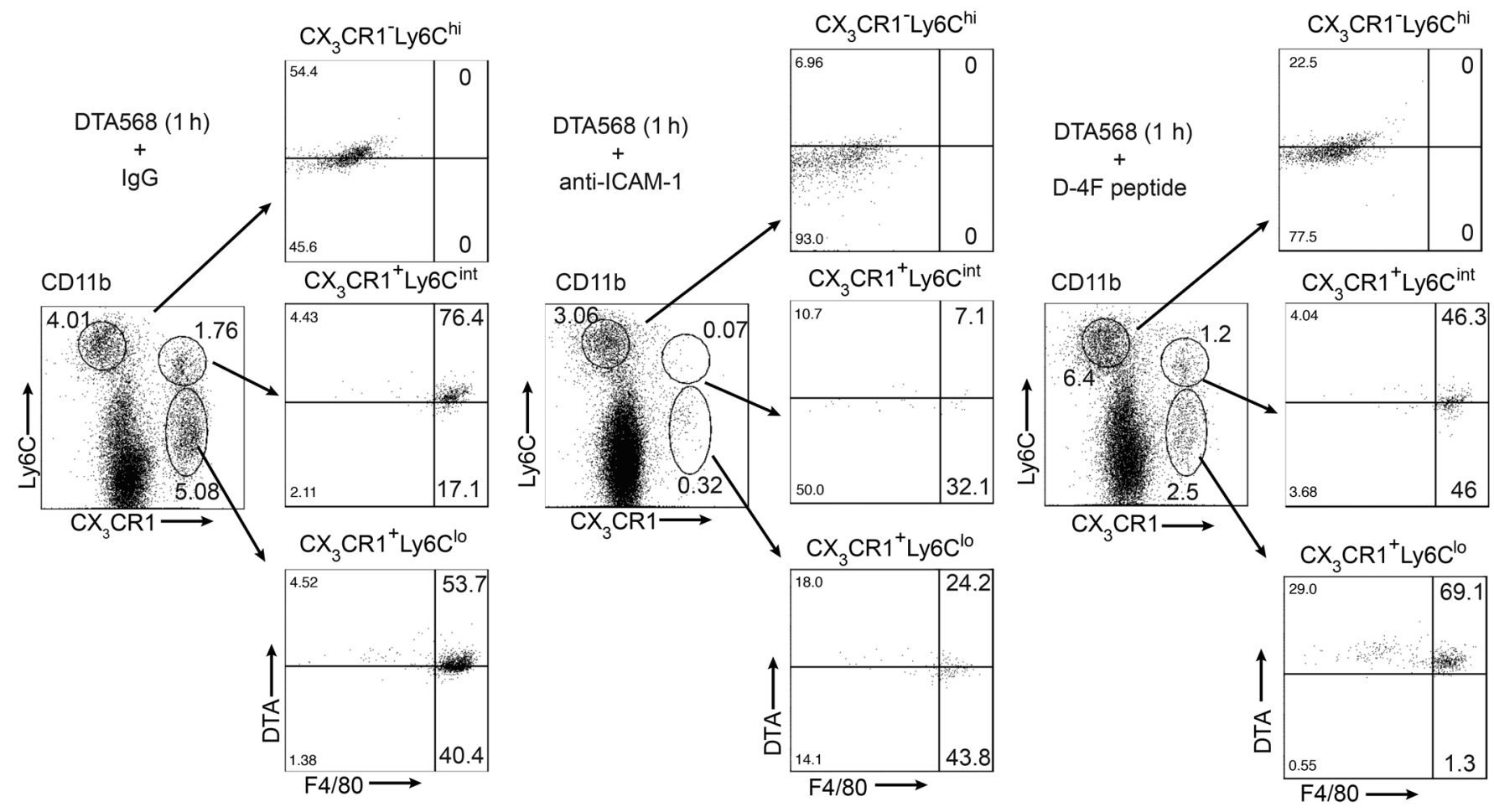

Figure 7. Contrast uptake by monocytes in the kidney. Mice were treated with CF568-labeled DTA for 1 hour before kidney leukocytes were sorted using CD11b and analyzed by flow cytometry. Monocytes/macrophages identified using CX CR1, Ly6C, and F4/80 were analyzed for DTA uptake. Inhibitor studies using anti-ICAM-1 and D-4F peptide in mice before DTA treatment were also analyzed using flow cytometry. Images are representative of 3 independent experiments.

Nevertheless, these data provide evidence that contrast administration activates the inflammasome and tubular injury early in humans undergoing coronary angiography.

\section{Discussion}

In this study, we elucidate the molecular and cellular mechanisms that drive AKI induced by radiographic contrast media. The pathogenesis of CI-AKI requires interplay among the intrarenal immune system, the renal tubular epithelium, and leukocyte recruitment that includes (a) uptake of contrast into perivascular resident and recruited renal phagocytes, (b) Nlrp3 inflammasome activation and IL-1-dependent leukocyte recruitment, (c) tubular reabsorption of contrast via renal DPEP-1, and (d) contrast-induced tubular injury (Figure 12). Each event is necessary, and none alone is sufficient to injure the kidney, transcending the notion that toxic effects of contrast agents on TECs alone are sufficient to drive the pathogenesis of CI-AKI. While tubular cell uptake of contrast and subsequent toxicity are critical for leukocyte recruitment and CI-AKI, the renal immune response plays an important role.

The toxicity of contrast agents on TECs may be overestimated by in vitro studies due to multiple confounding variables. The i.v. administration of contrast media, which is sufficient under specific clinical circumstances to induce AKI, is disseminated and largely diluted before reaching the kidney. Our data show that in euvolemic states, contrast is filtered and rapidly excreted in the urine, with very little tubular uptake. During volume depletion, however, contrast is reabsorbed and accumulates focally within 30-60 minutes in the tubules, where cellular toxicity likely then occurs. In combination with an inflammatory response, CI-AKI ensues. The finding of focal accumulation of contrast media in tubules sheds light mechanistically on the increased risk of CI-AKI in patients with volume depletion or reduced nephron number and CKD. These observations also explain the effectiveness of the only known therapeutic intervention for CI-AKI, prehydration with crystalloid solutions $(28,29)$.

Recent studies have demonstrated an essential role for resident renal phagocytes in regulating the immune response to circulating immune complexes $(9,10)$. While IL-1 and other proinflammatory cytokines were suggested to be important downstream effectors of renal inflammation regulated by these cells, the molecular pathways regulating IL-1 secretion were not identified (9). Our results further clarify the biology of resident renal phagocytes and infiltrating monocytes/macrophages, identifying the Nlrp3 inflammasome as a key innate sensor in these leukocytes. While resident renal phagocytes and infiltrating monocyte/macrophage populations were central in the innate immune response to contrast, neutrophils, on the other hand, while recruited to the kidney and contributing to injury, were not found to play a significant role in inflammasome activation. The mechanism of inflammasome activation by radiographic contrast agents in monocytes/macrophages is multifactorial but likely involves osmotic stress and reactive oxygen species induced directly by contrast, as well as indirect responses to DAMPs release from injured TECs $(1,30,31)$. Together with prior studies by our group and others, these data establish a two-compartment model of Nlrp3 function in the kidney $(11,13,16,32)$. The canonical Nlrp inflammasome is centered primarily in resident renal phagocytes 
A

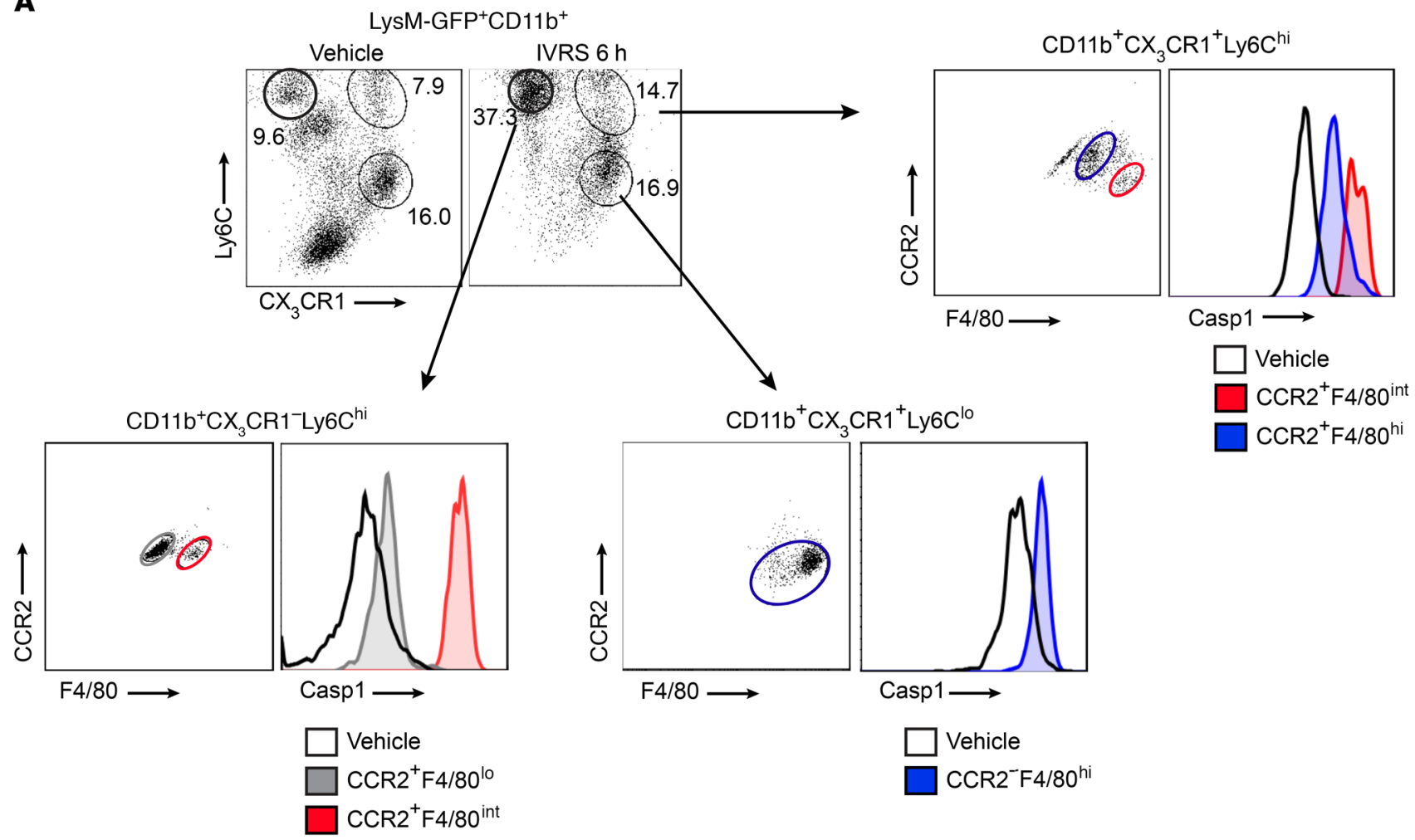

B

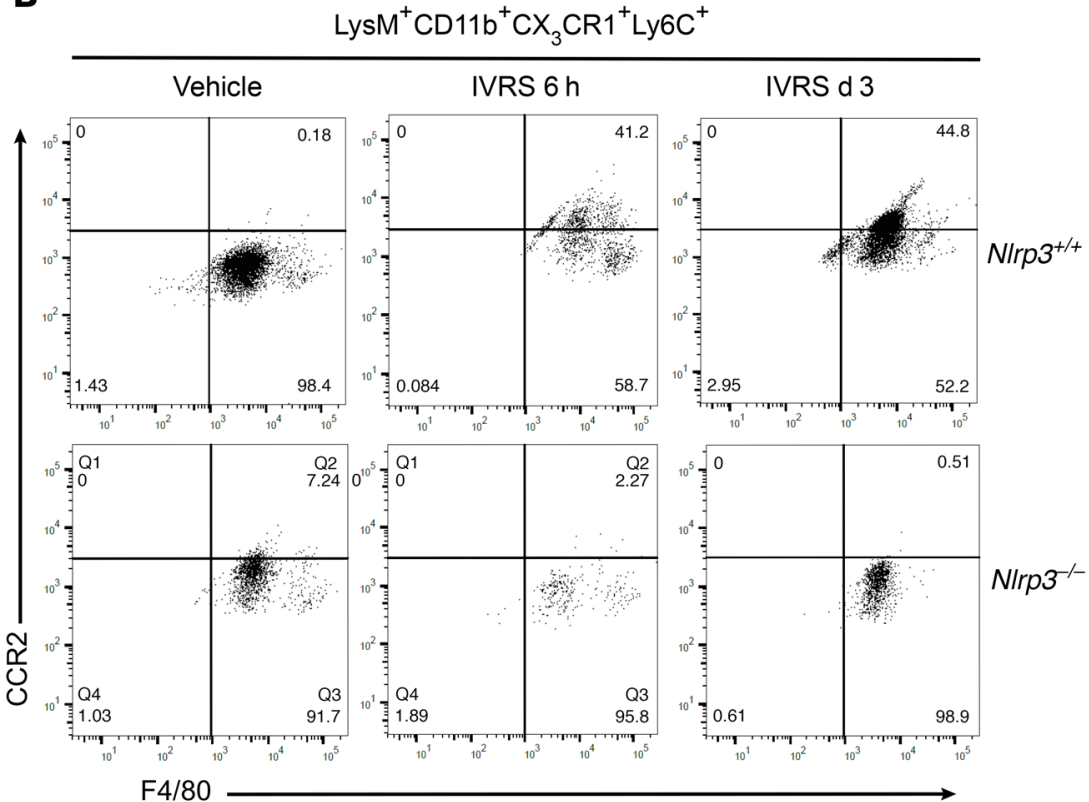

Figure 8. Characterization of inflammasome activation in infiltrating proinflammatory monocytes in Cl-AKI. Lys $\mathrm{M}^{(g f p / g f p)}$ and $\mathrm{NIrp3}^{-/-}$Lys $\mathrm{M}^{(g f / / g f p)}$ mice were treated with vehicle or ioversol and assessed at various time points. (A) LysM-GFP+ CD11b+ leukocytes were isolated from vehicle- or ioversol-treated $L y s M^{(g f p / g f p)}$ mice at 6 hours and analyzed for inflammasome activation (active caspase-1) in infiltrating monocytes and resident macrophages by flow cytometry using CX $C R 1$, Ly6C, $\mathrm{F} 4 / 80$, and CCR2. Images are representative of 3 independent experiments. (B) LysM-GFP+CD11b+CX CR1 $1^{+}$Ly6C ${ }^{+}$monocytes/macrophages were isolated

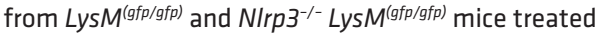
with vehicle or ioversol and analyzed at 6 hours or 3 days using CCR2 and F4/80. Images are representative of 3 independent experiments. and recruited monocytes/macrophages, while a noncanonical Nlrp3 exists in TECs to regulate apoptosis. Since contrast agents did not induce apoptosis in primary TECs, Nlrp3 function in CI-AKI is mainly canonical. Our data are consistent with the study by Linkermann and colleagues, who showed that neither apoptosis nor necroptosis plays a role in contrast-induced epithelial cell death (19). Our findings are also consistent with a recent study demonstrating a role for Nlrp3 in CI-AKI (33) but contradict the observation that the phenotype is due to Nlrp3-dependent regulation of epithelial cell apoptosis. Significant differences in methodology, such as the use of cell lines rather than primary cell cultures, likely explain these discrepant findings.

Using intravital microscopy we show monocyte/macrophage sampling of contrast transported from the urine via a direct interaction with tubular cells. This urinary immune surveillance was performed by recruited LysM-GFP ${ }^{+}$monocytes/macrophages as opposed to $\mathrm{CX}_{3} \mathrm{CR}^{+}$resident renal phagocytes that took up contrast primarily from the vascular space. The factors involved in the monocyte/macrophage and tubular cell "synapse" require further study. The uptake of contrast by infiltrating monocytes/ 
A
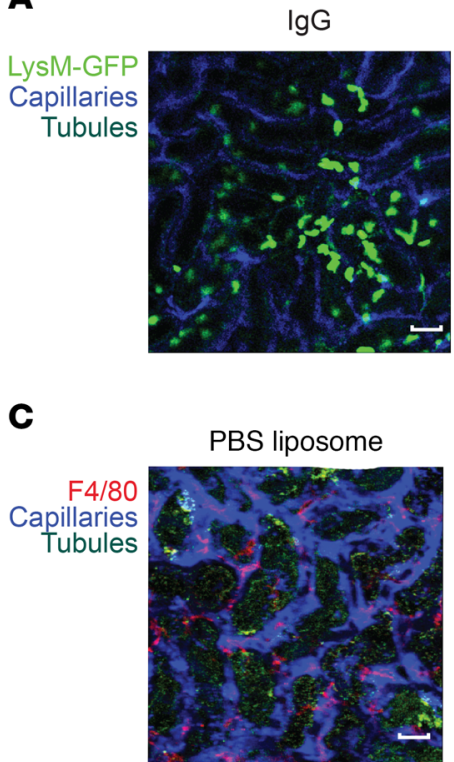

PBS liposome

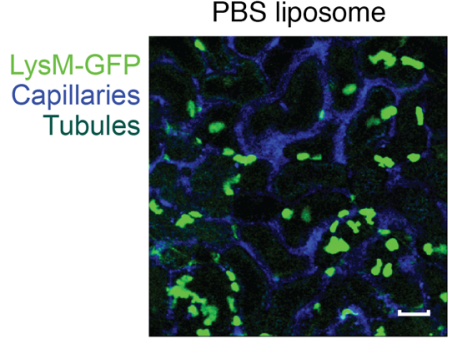

E

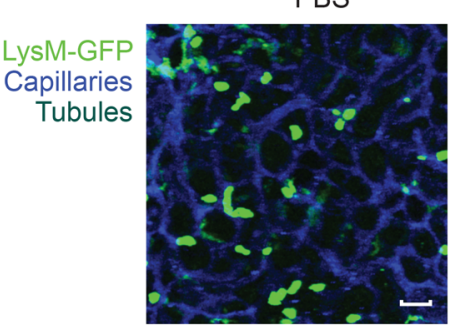

Anti-Ly6G

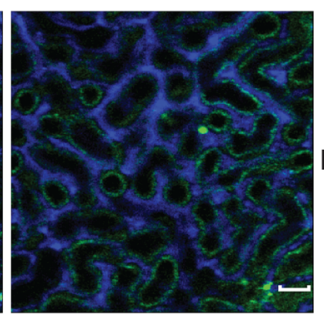

Clodronate-liposome

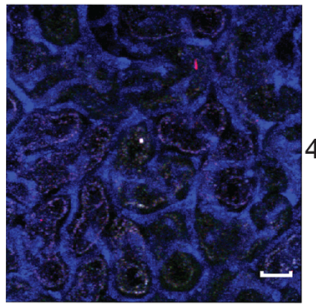

Clodronate-liposome

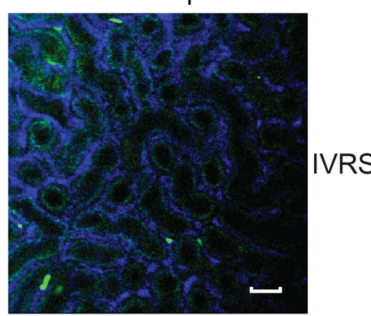

IL-1Ra

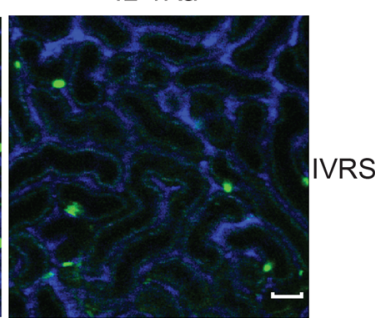

B

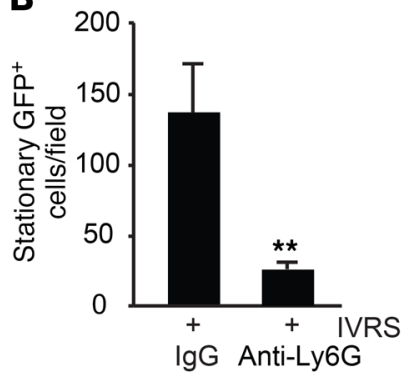

D
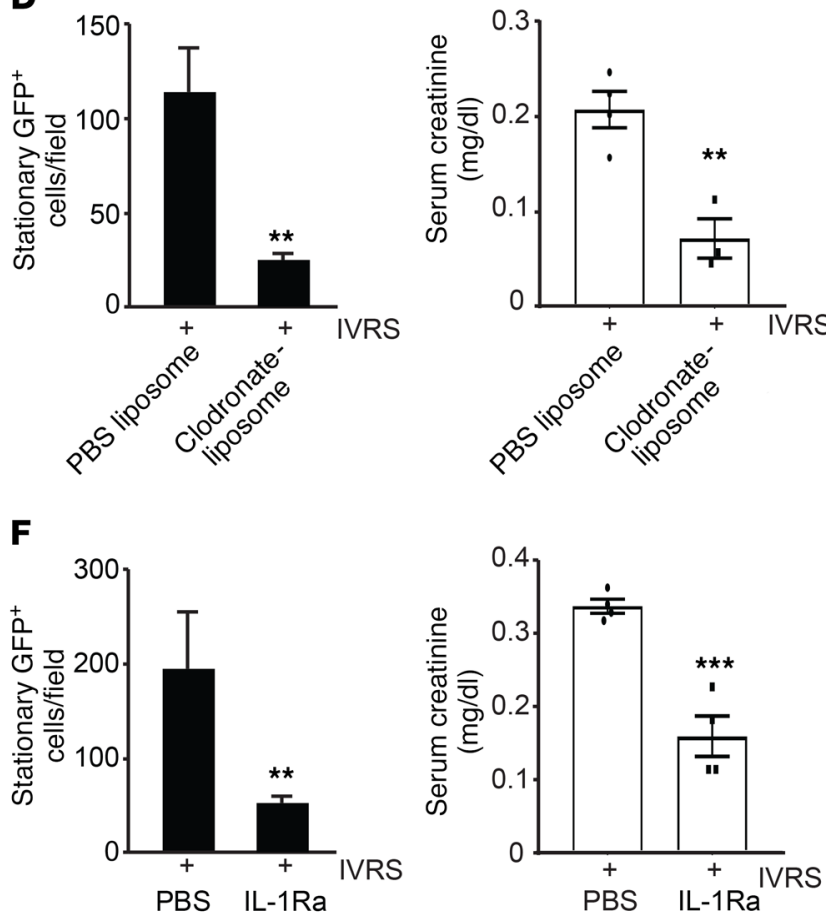

Figure 9. Role of resident phagocytes and leukocyte recruitment in CI-AKI. (A) Multiphoton intravital microscopy of leukocyte recruitment in LysM(gfp/gfp) mice at 6 hours following ioversol administration. Mice were pretreated with anti-Ly6C or anti-lgG isotype control. Capillaries were labeled with Qtracker and tubules are visualized by autofluorescence. Images are representative of at least 3 independent experiments. Scale bars: $100 \mu \mathrm{m}$. (B) Quantification of stationary LysM-GFP+ leukocytes at 6 hours (anti-Ly6G vs. anti-IgG isotype, ${ }^{*} P=0.004, n=3-5$ /group, 2-tailed Student's $t$ test) and serum creatinine at 3 days (ref. values $0.04-0.08 \mathrm{mg} / \mathrm{dl}$ ) ${ }^{* * *} P=0.002, n=4 /$ group, 2 -tailed Student's $t$ test). (C) Multiphoton intravital microscopy of leukocyte recruitment in $L y s M^{(g f / g f p)}$ mice at 6 hours following ioversol administration. Resident renal phagocytes were depleted from $L y s M^{(g f p / g f p)}$ mice using clodronate-liposomes, while PBS liposomes were used as control (lower panels). Depletion was confirmed by immunofluorescence for F4/80 at 48 hours (upper panels). Images are representative of at least 3 independent experiments. Scale bars: $100 \mu \mathrm{m}$. (D) Quantification of stationary LysM-GFP+ leukocytes at 6 hours (clodronatevs. PBS liposomes, ${ }^{* *} P=0.006, n=3-5 /$ group, 2-tailed Student's $t$ test) and serum creatinine at 3 days ( ${ }^{* *} P=0.005, n=3-4 /$ group, 2 -tailed Student's $t$ test). (E) Multiphoton intravital microscopy of leukocyte recruitment in $L y s M^{(g f p / g f p)}$ mice at 6 hours following ioversol administration. IL-1 was blocked using IL-1Ra. Images are representative of at least 3 independent experiments. Scale bars: $100 \mu \mathrm{m}$. (F) Quantification of stationary LysM-GFP+ leukocytes (IL-1Ra vs. PBS, ${ }^{* *} P=0.0009, n=4 /$ group, 2-tailed Student's $t$ test) and serum creatinine on day 3 ( ${ }^{* * *} P=0.0002, n=4-5$ /group, 2-tailed Student's $t$ test).

macrophages depended on the cell adhesion molecule ICAM-1. SR-A did not appear to play a role; however, other scavenger receptors may be involved. On the contrary, neutrophils did not take up measurable amounts of contrast in vivo, perhaps reflecting differential expression of cell surface receptors needed for this function compared with monocyte/macrophages. Therefore, the kidney possesses several mechanisms to monitor the vascular and urinary environments for potential pathogen-associated molecular patterns (PAMPs) and DAMPs. While it seems clear that resident renal phagocytes perform an important sentinel function to trigger immune responses in the kidney, our data suggest that urinary immune surveillance by recruited monocytes/macrophages may serve to amplify the inflammatory response via the uptake of DAMPs and the activation of innate immune pathways such as 
the Nlrp3 inflammasome. In a different context, urinary immune surveillance may also represent an important mechanism for antigen uptake and the development of adaptive immunity. Future investigation into the relationship between vascular and urinary immune surveillance and the role of luminal tubular receptors such as DPEP-1 in this process will only continue to increase the understanding of renal inflammation, kidney injury, and disease.

Finally, our studies identify several new therapeutic targets to prevent CI-AKI in humans. First, pilot data in our human CI-AKI cohort confirm the early activation of inflammasomerelated pathways and inflammation in patients who receive contrast during coronary angiography. Targeting IL-1, the Nlrp3 inflammasome, or leukocyte recruitment may be useful as adjunct to current standard of care for the prevention of CI-AKI. Second, we identify renal DPEP-1 as the receptor for the tubular reabsorption of contrast. The DPEP-1 inhibitor cilastatin is currently used clinically to prevent tubular uptake and metabolism of the antibiotic imipenem (34) and would be immediately available as a single agent for therapeutic clinical trials in CI-AKI. Recently, a study by Hori et al. suggested that antibiotic nephrotoxicity and the beneficial effects of cilastatin were mediated by the megalin receptor in the proximal tubule (26). While this may contribute to tubular contrast uptake, our in vitro data clearly show a role for DPEP-1 in this process. Whether cilastatin also provides beneficial off-target effects in CI-AKI will require further study. While preventing tubular uptake of contrast with i.v. fluids is the key first step to interrupting the pathogenesis of CI-AKI, in patients who are unable to receive i.v. fluids, targeting inflammation or DPEP-1 becomes a feasible option.

\section{Methods}

\section{Mouse studies}

Nlrp $3^{+/+}$(C57BL/6, The Jackson Laboratory), Nlrp3 $3^{--}$(provided by J. Tschopp, University of Lausanne, Lausanne, Switzerland), Lys $M^{(g f / g f p)}$ (provided by T. Graf, Centre for Genomic Regulation, Barcelona, Spain), $C X_{3} C R 1^{(s f /+)}$ (The Jackson Laboratory), and CD11c-DTR ${ }^{(\alpha f /+)}$ (The Jackson Laboratory) mice were on a C57BL/6 background and housed under standard conditions. Nlrp $3^{-/-} L y s M^{(g f / g f p)}$ and $N \operatorname{lrp} 3^{+/+} L y s M^{(g f p / g f p)}$ littermates were generated by crossing $L y s M^{(g f / g f p)}$ and $N l r p 3^{--}$mice. $\mathrm{Nlrp}^{+/+}$and $\mathrm{Nlrp} 3^{-/-}$littermates were also generated and used in experiments. Mice were used between 8 and 12 weeks of age. For induction of CI-AKI, mice underwent water deprivation for 2 days prior to i.v. administration ( $5 \mathrm{ml} / \mathrm{kg}$ body weight) of ioversol (Optiray 320, Mallinckrodt Pharmaceuticals) or DTA (Sigma-Aldrich). For hydration studies, mice did not undergo water deprivation and were given $200 \mu \mathrm{l}$ normal saline i.v. prior to contrast. Depletion studies in mice were completed using clodronate-liposomes (200 $\mu$ l i.v., ClodronateLiposomes.com), PBS liposomes (200 $\mu$ li.v., ClodronateLiposomes.com), anti-Ly6G (200 $\mu \mathrm{g}$ i.p., BioXCell, catalog BE0075-1), anti-ICAM-1 (200 $\mu$ g i.p., BioXCell, catalog BE0020-1), anti-IgG (200 $\mu$ g i.p., BioXCell, catalog BE0086), D-4F peptide $(21,22)$ (20 $\mu$ g i.p., CanPeptide), and Kineret $(100 \mathrm{mg} /$ $\mathrm{kg}$ i.p., Sobi). CD11c-DTR ${ }^{(g f /+)}$ mice were depleted via diphtheria toxin (8 ng/g body weight i.p.). Cilastatin (Tocris Bioscience) was administered at a dose of $25 \mathrm{mg} / \mathrm{kg}$ in $100 \mu \mathrm{l}$ normal saline 30 minutes prior to contrast injection (control mice only received $100 \mu \mathrm{l}$ saline). Urine was collected using metabolic cages. For chimera studies, bone marrow cells were isolated from $\mathrm{Nlpr}^{+/+}$and $\mathrm{Nlpp}^{-/-}$mice and administered to clodronate-liposome-treated and irradiated recipients as described previously (14). Depletion of circulating and resident immune cells in the kidney was verified using intravital microscopy.

\section{Cell culture}

Murine TECs. TECs were isolated from kidneys harvested from mice between the ages of 8 and 12 weeks. Under sterile conditions, the kidney capsule and surrounding fatty tissue were removed from the kidney, and the renal cortex was removed. Renal cortical tissue was incubated in $1.5 \mathrm{mg} / \mathrm{ml}$ collagenase (Sigma-Aldrich) and incubated at $37^{\circ} \mathrm{C}$ for 1 hour. Tissue was passed through a $40-\mu \mathrm{M}$ nylon cell strainer (BD Biosciences) and washed with HBSS (Gibco, Thermo Fisher Scientific). Cells were plated on collagen IV-coated (SigmaAldrich) culture dishes and grown in DMEM/F-12 (Gibco) containing 10\% FBS, $125 \mathrm{ng} / \mathrm{ml}$ prostaglandin E1, $10 \mathrm{ng} / \mathrm{ml} \mathrm{EGF,} 1.8 \mu \mathrm{g} / \mathrm{ml}$ L-thyroxine, $3.38 \mathrm{ng} / \mathrm{ml}$ hydrocortisone, $2.5 \mathrm{mg} / \mathrm{ml}$ insulin-transferrinsodium selenite supplement, and $25 \mathrm{ng} / \mathrm{ml}$ murine EGF (SigmaAldrich) at $37^{\circ} \mathrm{C}$. Cells were washed the next day and grown to confluence in $\mathrm{K} 1$ media at $37^{\circ} \mathrm{C}, 5 \% \mathrm{CO}_{2}$ humidified incubator. Renal TECs isolated from Rip $^{-/-}$mice were prepared as above by Anthony M. Jevnikar of the University of Western Ontario (London, Ontario, Canada). All in vitro experiments were completed within two cell passages.

HPTCs and glomerular endothelial cells. Kidney tissues were obtained from non-diseased margins of human nephrectomies that were performed for medically indicated reasons. Kidney tissues were collected within 2 hours of surgery in HBSS supplemented with $5 \mathrm{mg}$ / $\mathrm{ml}$ penicillin/streptomycin on ice. Following removal of the renal capsule, cortical tissue was carefully cut away and minced using aseptic techniques. After digestion in collagenase $(1.5 \mathrm{mg} / \mathrm{ml}$ in HBSS $)$ at $37^{\circ} \mathrm{C}$ for 60 minutes, samples were passed through serial filters from 200 to $45 \mu \mathrm{m}$ to remove intact glomeruli and large cellular debris. Samples were then plated on plastic cell culture plates at $37^{\circ} \mathrm{C}$ for 90 minutes in $\mathrm{K} 1$ culture medium DMEM/F12 containing 10\% FBS, 1\% penicillin-streptomycin, $125 \mathrm{ng} / \mathrm{ml}$ prostaglandin $\mathrm{E} 1,1.8 \mu \mathrm{g} / \mathrm{ml}$ L-thyroxine, $3.38 \mathrm{ng} / \mathrm{ml}$ hydrocortisone, $2.5 \mathrm{mg} / \mathrm{ml}$ insulin-transferrinsodium selenite supplement, and $10 \mathrm{ng} / \mathrm{ml} \mathrm{EGF} \mathrm{(Sigma-Aldrich).}$ HPTCs were collected and plated onto collagen IV-coated cell culture plates. To maintain the epithelial phenotype, HPTCs were used at fewer than 2 passages. For cell toxicity studies, necrosulfonamide $(10 \mu \mathrm{M}$, Calbiochem), Necrostatin-1 (10 $\mu \mathrm{m}$, Sigma-Aldrich), or Ferrostatin-1 (10 $\mu \mathrm{M}$, Sigma-Aldrich) was applied to cells 30 minutes prior to contrast agents. Primary cultures of human glomerular endothelial cells (GECs; Cell Systems) were commercially obtained and cultured according to the manufacturer's protocol.

Murine BMDMs. BMDMs were isolated from femurs and tibiae of mice aged 8-12 weeks. Bone marrow was isolated on ice and washed with HBSS twice. BMDMs were plated on culture dishes and grown in RPMI-1640 (Gibco) supplemented with L929 conditioned media and penicillin/streptomycin for 7 days before use in experiments. BMDMs were primed with $200 \mathrm{ng} / \mathrm{ml}$ Ultrapure LPS (Sigma-Aldrich) for 4 hours before treatments.

Murine renal pericyte isolation and culture. Pericytes were isolated from kidneys of mice aged 8-12 weeks as previously described (35). Briefly, pericytes were isolated using magnetic bead isolation (MagCellect Ferrofluid, R\&D Systems) and anti-PDGFR $\beta$-positive (clone 28E1, Cell Signaling Technology) selection. Pericytes were grown on 
A

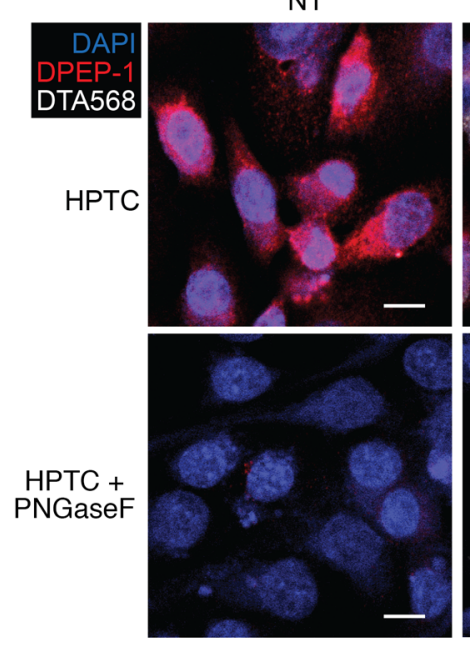

C

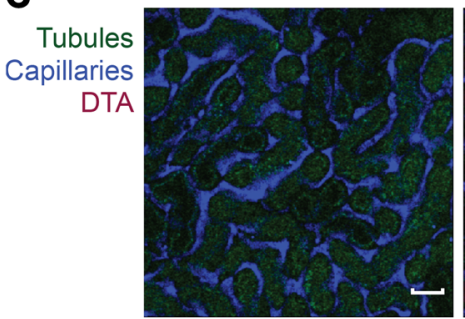

DTA568

Before

D

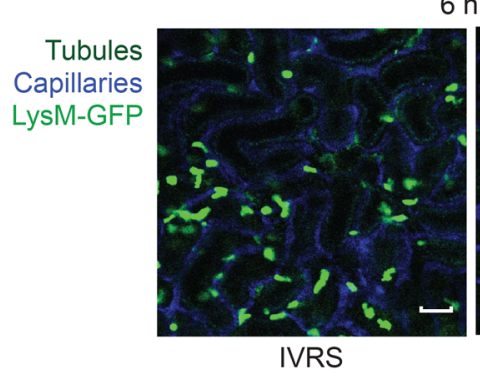

F

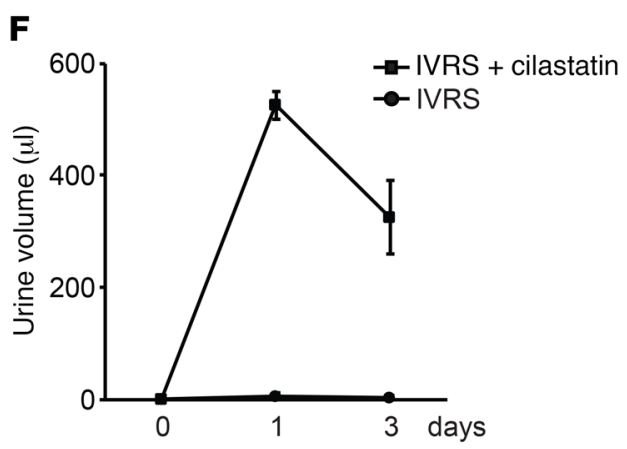

DTA568
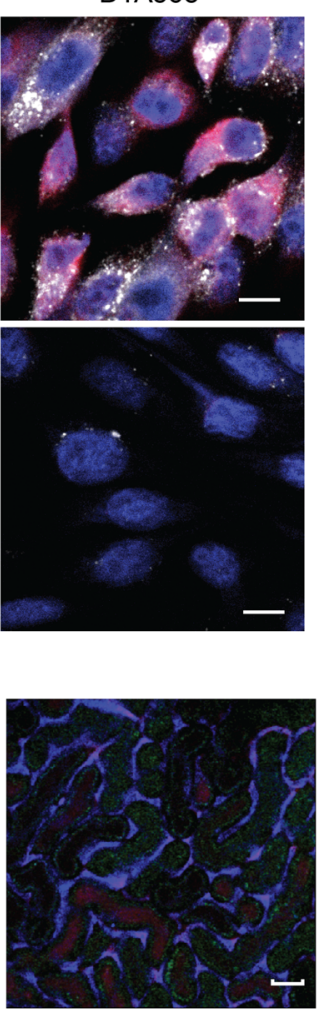

$1 \mathrm{~min}$
B
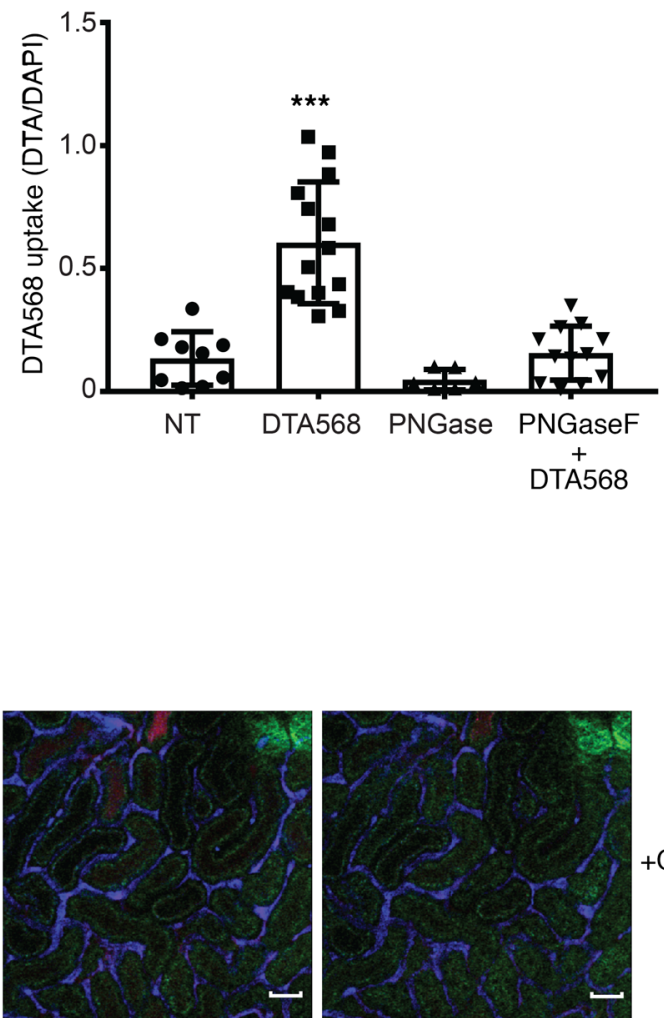

$20 \min$

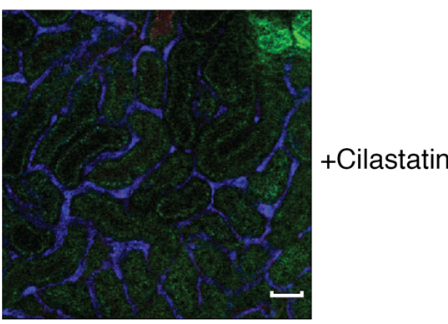

$40 \mathrm{~min}$

E

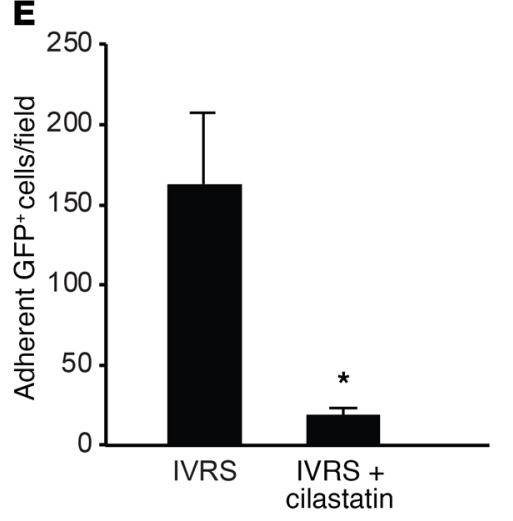

G

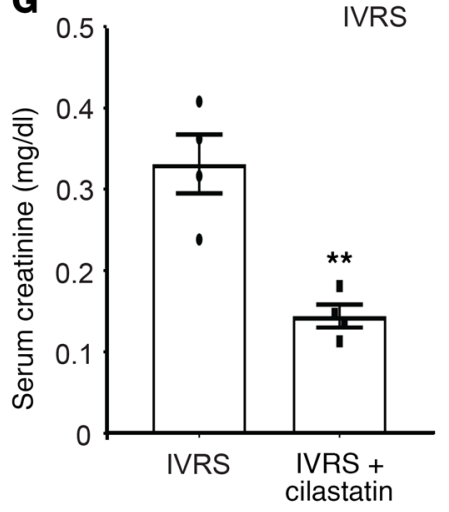


Figure 10. Renal DPEP-1 mediates tubular uptake of contrast from the urine. (A and B) Contrast uptake (internalization) was imaged and quantified in human renal proximal TECs (HPTCs) using CF568-labeled DTA. PNGaseF was used to de-glycosylate DPEP-1 on HPTCs as a negative control. Scale bars: $10 \mu \mathrm{m}$. NT vs. CF568-DTA, ${ }^{* *} P=0.001, n=9-14 /$ group, ANOVA. (C) WT mice were treated with cilastatin prior to administration of CF568-DTA, and kidneys were imaged by multiphoton intravital microscopy over 40 minutes. Capillaries were labeled with Qtracker, and tubules are visualized by autofluorescence. Images are representative of 3 independent experiments. Scale bars: $100 \mu \mathrm{m}$. (D and $\mathbf{E}) L y s M^{(g f f / g f p)}$ mice were treated with cilastatin $(25 \mathrm{mg} / \mathrm{kg})$ or vehicle control prior to administration of ioversol, and kidneys were imaged by multiphoton intravital microscopy. Scale bars: $100 \mu \mathrm{m}$. Inflammation was quantified by manual counting of stationary GFP+ leukocytes (saline vs. cilastatin, ${ }^{*} P=0.02, n=3$ /group, 2-tailed Student's $t$ test). (F and G) WT C57BL/6 mice were treated with cilastatin or normal saline vehicle prior to ioversol administration. Urine output and serum creatinine as a measure of kidney function was determined on days 1 and 3 after contrast treatment (ref. values $0.04-0.08 \mathrm{mg}$ / dl) (saline vs. cilastatin, creatinine day $3,{ }^{* *} P=0.003, n=4 /$ group, 2 -tailed Student's $t$ test).

gelatin-coated culture dishes in pericyte medium. Cells were characterized by the positive marker PDGFR $\beta$ (clone C82A3, Cell Signaling Technology) and negative marker E-cadherin (clone G10181, BD Biosciences). Renal pericytes (NG2+) were also characterized in vivo via intravital microscopy of $\mathrm{Tg}(\mathrm{Cspg} 4-\mathrm{DsRed}$.T1)1AkikJ mice (The Jackson Laboratory).

THP-1 cell line. The human myelogenous leukemia THP-1 cell line was purchased from ATCC. THP-1 cells were cultured in RMP1-1640 supplemented with $10 \%$ FBS, $1 \%$ penicillin/streptomycin, $0.05 \mathrm{mM}$ $\beta$-mercaptoethanol, and $1 \mathrm{mM}$ sodium pyruvate. Cells were maintained in suspension in a $37^{\circ} \mathrm{C}, 5 \% \mathrm{CO}_{2}$ humidified incubator. Cells were differentiated with $100 \mathrm{nM}$ PMA (Sigma-Aldrich) for 24 hours prior to experiments.

Generation of necrotic DAMPs. DAMPs were prepared from HPTCs and THP-1 cells via treatment with hydrogen peroxide $(500 \mathrm{mM}$, 3 hours), nigericin ( $50 \mu \mathrm{M}, 30$ minutes), or ioversol (3 hours) before replacement with serum-free media for 24 hours to generate DAMPs. Supernatants were collected from treated cells and added to new THP- 1 cultures for 6 hours. Necrotic DNA was generated via filtration of necrotic cell debris by a QIAshredder homogenizer (QIAGEN).

\section{CRISPR/Cas9 NLRP3 gene editing}

Nlrp3 $3^{--}$THP-1 cells were produced using the lentiCRISPRv2 shuttle plasmid (Addgene plasmid 52961, provided by Feng Zhang, Broad Institute and Massachusetts Institute of Technology, Boston, Massachusetts, USA). The lentiCRISPRv2 included 3 expression cassettes, the Cas9 nuclease, puromycin resistance, and the guide RNA scaffold, into which a pair of annealed oligonucleotides complementary to bases 767-787 in the first exon of the Nlrp3 gene were cloned using BsmI digestion sites. The following Nlrp3 targeting oligonucleotides were synthesized: 5'-CACCGCTGCAAGCTGGCCAGGTACC-3', 5'-AAACGGTACCTGGCCAGCTTGCAGC-3'. Oligonucleotides were annealed by the addition of $0.1 \mathrm{nmol}$ of each to $0.5 \mu$ polynucleotide kinase in 10× T4 ligation buffer (New England BioLabs) at $37^{\circ} \mathrm{C}$ for 30 minutes, $95^{\circ} \mathrm{C}$ for 5 minutes, followed by ramping down the temperature by $5^{\circ} \mathrm{C} / \mathrm{min}$ to $25^{\circ} \mathrm{C}$. The lentiCRISPRv2 plasmid was then digested with $\mathrm{BsmBI}$ and dephosphorylated with alkaline phosphatase for 30 minutes at $37^{\circ} \mathrm{C}$, and the annealed oligonu- cleotides were ligated into the BsmBI-digested lentiCRISPRv2. HEK293T cells were transfected with the Nlrp3-targeting lentiCRISPRv2 construct, viral envelope plasmid pCMV-VSVG, and viral packing vector psPAX2. The virus-containing medium was collected every 12 hours and concentrated by ultracentrifugation at 50,000 $\mathrm{g}$ for 120 minutes. THP-1 cells were transduced with $5 \mu$ l concentrated viral particles and maintained at $37^{\circ} \mathrm{C}, 5 \% \mathrm{CO}_{2}$ in complete RPMI containing $5 \mu \mathrm{g} / \mathrm{ml}$ puromycin for a total of 8 passages. To confirm the effectiveness of the CRISPR gene editing, genomic DNA was isolated from THP-1 cells. PCR for amplification of the target cleavage site was performed using the following primers: F: 5'-TCGAGCCAACAGGAGAACTT-3', R: 5'-TCGGCTCATCTCTTTTTGCT-3'. PCR products were digested with KpnI. WT THP-1 cells showed a 564and 241-bp fragment, whereas $\mathrm{Nlrp}^{-/-}$cells showed an 805-bp band before and after KpnI digestion, indicating that $\mathrm{Nrp3}^{-/-}$(CRISPR) cells had incorporated the appropriate mutation within the Nlrp3 gene. Nlrp3 protein expression was determined by immunoblotting as described below (Supplemental Figure 16).

\section{Immunoblotting}

Protein samples were separated by SDS-PAGE gels under reducing conditions. Proteins were transferred onto nitrocellulose membranes (GE Healthcare) and blocked for 1 hour with blocking solution before overnight incubation at $4^{\circ} \mathrm{C}$ with primary antibody. Blots were washed and incubated with appropriate secondary antibody conjugated to horseradish peroxidase at room temperature. Proteins were visualized with ECL Western blotting detection reagents (GE Healthcare) and digitally captured with a ChemiDoc MP device (Bio-Rad). Densitometry was performed using Image Lab software (Bio-Rad) and normalized as indicated. Antibodies used were as follows: caspase- 8 (clone 1C12, Cell Signaling Technology), caspase-3 (Cell Signaling Technology, catalog 9662), PARP (Cell Signaling Technology, cata$\log$ 9542), MLKL (clone D6H3V, Cell Signaling Technology), RIPK3 (clone E1Z1D, Cell Signaling Technology), caspase-1 (Santa Cruz Biotechnology Inc., catalog SC-622 targeting N-terminal CARD domain), Nlrp3 (clone CRYO-2, Adipogen), ASC (Adipogen, catalog AL177), IL-1 $\beta$ (pro-IL1 $\beta$ : clone 3A6; cleaved-IL-1 $\beta$ : clone D3A3Z, Cell Signaling Technology), GAPDH (Abcam, catalog ab9485), and $\beta$-tubulin (SigmaAldrich, catalog T0198).

\section{ELISA}

THP-1 cells were differentiated with PMA, and BMDMs were primed with ultrapure LPS for 4 hours before treatment. Nigericin $(50 \mu \mathrm{M}$, Tocris Bioscience) or ATP (5 mM, Sigma-Aldrich) treatment was used as positive controls. Cell supernatants were used to detect IL-1 $\beta$ cytokine secretion by ELISA (BD Biosciences) as per the manufacturer's instructions.

\section{MTT assay}

TECs were assessed for cell viability using MTT assay as per the manufacturer's instructions (Invitrogen, Thermo Fisher Scientific). TECs were grown to monolayer and treated with various reagents for 24 hours in serum-free media. Cells were incubated with MTT (12 mM) for 4 hours at $37^{\circ} \mathrm{C}$. Viable cells converted MTT to formazan, which was solubilized by DMSO before quantification by spectrophotometry at $540 \mathrm{~nm}$. Cell viability was calculated for each treatment relative to untreated cells. 
A

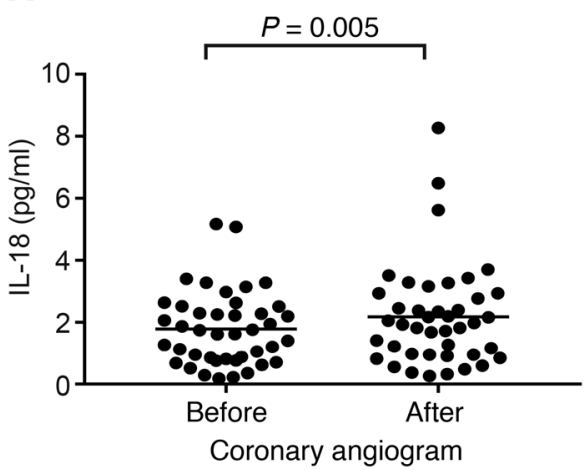

B

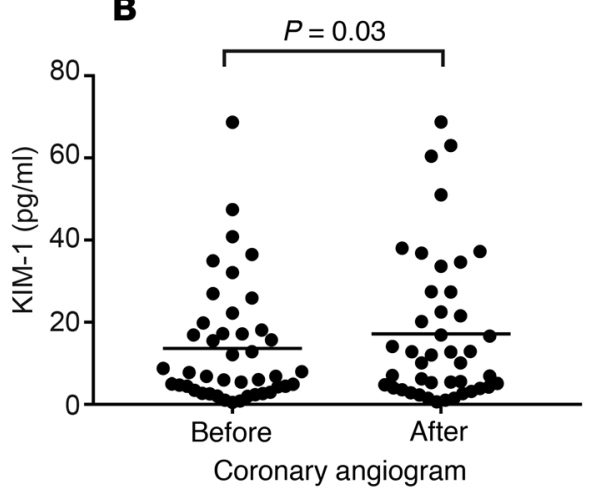

C

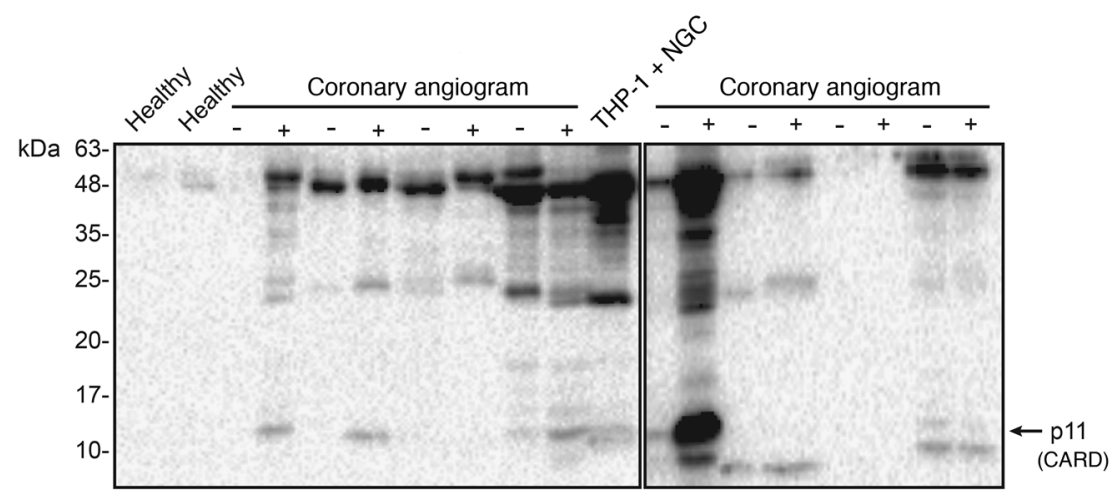

IB: caspase-1

D

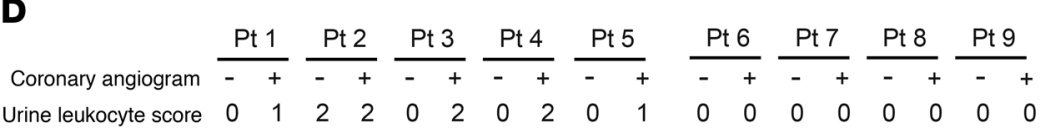

$$
\mathrm{kDa} 48
$$

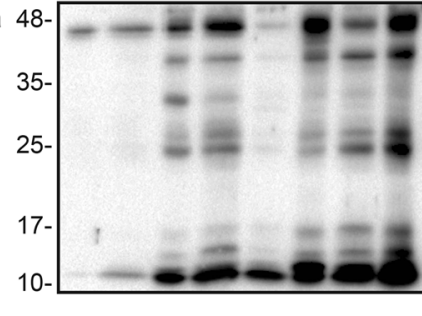

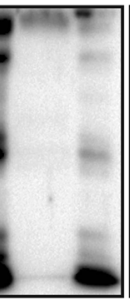

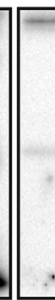

IB: caspase-1<smiles>O=[W]=O</smiles>

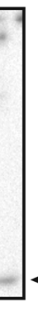

$\leftarrow$ p 11
E

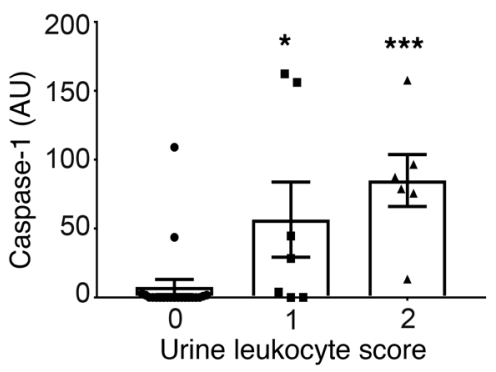

Figure 11. Inflammasome-related biomarkers are detected in human urine following contrast administration. (A) Urinary IL-18 and (B) KIM-1 before and less than 24 hours after coronary angiogram as determined by Meso Scale Discovery assay. Data points normalized to urine creatinine are shown (IL-18 before vs. after, $P=0.005, n=42 /$ group; KIM-1 before vs. after, $P=0.03, n=42 /$ group, Wilcoxon's signed-rank test). (C) Caspase- 1 immunoblotting from urine pellets in 2 healthy control individuals and 8 patients before and less than 24 hours after coronary angiogram. Nigericin-treated THP- 1 macrophages were used as a positive control. Arrow indicates cleaved capase-1 N-terminal CARD domain. (D) Caspase- 1 immunoblotting from urine pellets in 9 patients before and less than 24 hours after coronary angiogram, with corresponding urine leukocyte score. (E) Quantification of caspase-1 expression versus leukocyte score in 17 patient urine samples before and less than 24 hours after coronary angiogram (caspase-1 densitometry normalized to urine creatinine per urine leukocyte score, 0 vs. $1:{ }^{*} P=0.011,0$ vs. $2:{ }^{* *} P=0.0001, n=21,7$, and 6 /group, ANOVA).

\section{Live cell imaging}

Cell death assay. TECs were grown to monolayer and treated with various reagents for 24 hours in color-free, serum-free media in the presence of propidium iodide (BD Biosciences) to label necrotic cells. TECs were imaged hourly for 24 hours using an automated fluorescence microscope (IncuCyte, Essen BioScience) in a $37^{\circ} \mathrm{C}, 5 \% \mathrm{CO}_{2}$ humidified incubator. Necrotic cell death was calculated by automated software (IncuCyte Zoom, Essen BioScience) as indicated by propidium iodide-positive cells.

Lipid peroxidation assay. TECs were grown to monolayer and treated with various reagents for 24 hours in color-free, serum-free media. Cumene hydroperoxide (100 $\mu \mathrm{M}$, Sigma-Aldrich) was used as a positive control for lipid peroxidation in TECs. Lipid peroxidation was measured using an Image-iT Lipid Peroxidation Kit (Life Technologies), which emits fluorescence in the presence of oxidation. TECs were imaged hourly for 24 hours using an automated fluorescence microscope (IncuCyte) in a $37^{\circ} \mathrm{C}, 5 \% \mathrm{CO}_{2}$ humidified incubator. Peroxidation was calculated by automated software (IncuCyte Zoom).

Contrast uptake in COS-1 cells and HPTCs. COS-1 (ATCC) cells were transfected with the pcDNA3.1-DPEP-1, human DPEP-1 expression plasmid using PolyJet In Vitro Transfection Reagent (SignaGen Laboratories) according to the manufacturer's protocol. After 48 
hours, cells were treated with CF568-labeled DTA for 30 minutes, and extracellular DTA was washed away before labeling with anti-DPEP-1 antibody (Proteintech, catalog 12222-1-AP). Similarly, HPTCs were also treated with CF568-labeled DTA. As a negative control, PNGaseF (New England BioLabs) was used to de-glycosylate DPEP-1, which alters its apical targeting and enzymatic activity (27). Image acquisition of $Z$-stacks (15- $\mu \mathrm{M}$ stack, $1.5-\mu \mathrm{M}$ steps) was done using a Nikon A1R $\mathrm{MP}+$ confocal microscope. Contrast uptake in cells was quantified using ImageJ software (NIH).

\section{Real-time PCR}

Murine kidney tissue was homogenized and total RNA was isolated using QIAshredder and RNeasy Mini Kit (QIAGEN) as per the manufacturer's instructions. cDNA was generated using M-MLV reverse transcriptase (Invitrogen) as per the manufacturer's instructions. The sequences for human KIM-1 primers and probes are as follows: F: 5'-CGTGGCTATCACCAGGTACATACT-3'， R: 5'-GCGTTCTGCAAAGCTTCAATC-3', probe FAM-5'-TCTCTAAGCGTGGTTGC-3'-MBG. The 18 S rRNA FAM/MGB probe was used as endogenous control. Real-time PCR reactions were made using Taqman Universal PCR Mastermix (Bio-Rad), primers (900 nM), probe (250 $\mathrm{nM})$, and cDNA template. Amplification was performed using the 7900HT Fast Real-Time PCR System (Bio-Rad).

\section{Immunofluorescence microscopy of fixed samples}

In vitro. THP-1 cells were differentiated with PMA on coverslips and treated with various reagents. Cells were incubated with FAM-YVADfmk (FLICA-caspase-1) (ImmunoChemistry Technologies) for 1 hour before fixation with $4 \%$ paraformaldehyde. Incubation with $\mathrm{NH}_{4} \mathrm{Cl}$ $(50 \mu \mathrm{M})$ was used to quench autofluorescence before permeabilization with $0.1 \%$ Triton X-100. Cells were blocked in 3\% BSA before incubation with primary antibodies overnight at $4^{\circ} \mathrm{C}$. Samples were then incubated with anti-human ASC (clone AL177, Adipogen), and cells were washed in PBS before incubation in secondary fluorescent antibody. Coverslips were mounted onto slides in ProLong Gold antifade (Invitrogen) containing DAPI. Images were acquired with a confocal fluorescence microscope (Olympus IX-70) with FluoView 1000 software (Olympus).

In vivo. Murine kidneys were fixed in 10\% neutral-buffered formalin and paraffinized before being mounted onto slides. Tissue sections were deparaffinized, blocked, and labeled with the following antibodies: anti-mouse KIM-1 (R\&D Systems, catalog MAB31817), fluoresceinlabeled Lotus tetragonolobus lectin (LTL, Vector Laboratories), anti-Ecadherin (clone G10181, BD Biosciences), or anti-F4/80 (clone CI:A3-1, Abcam). Images were acquired with a confocal fluorescence microscope (Olympus IX-70) with FluoView 1000 software. Quantification of F4/80 labeling was done using ImageJ software.

\section{Flow cytometry}

Murine kidney tissue was isolated from mice after perfusion via saline injection into the heart. Kidney capsule and fat were removed before incubation of whole tissue in $2 \mathrm{mg} / \mathrm{ml}$ collagenase at $37^{\circ} \mathrm{C}$ for $30 \mathrm{~min}$ utes. Tissue homogenate was then passed through a $40-\mu \mathrm{M}$ nylon cell strainer and washed in HBSS. Cell pellet was incubated in ACK lysis buffer (Gibco) for 3 minutes to remove red blood cells and washed in HBSS. Viable cells were isolated using a density gradient by mixing cells with Percoll (GE Healthcare) and centrifuging at $800 \mathrm{~g}$ for 25 minutes. Cells were washed in PBS and labeled with the following antibodies: Pacific Blue-conjugated anti-Ly6C (clone HK1.4, BioLegend), PE-conjugated anti-Ly6G (clone 1A8, BioLegend), PE/Cy5-conjugated anti-F4/80 (clone BM8, BioLegend), eFluor780-conjugated antiCD11b (clone M1/70, eBioscience), APC/Cy7-conjugated anti-CD11c (clone N418, BioLegend), FITC-conjugated CD45 (clone 30-F11, eBioscience), Pacific Blue-conjugated I-A/I-E (clone M5/114.15.2, BioLegend), PE-conjugated CD192 (CCR2) (clone SA203G11, BioLegend), Brilliant Violet 421-conjugated anti-CX $\mathrm{CR}_{3}$ (clone SA011F11, BioLegend), and FLICA caspase-1 (FAM-YVAD-fmk, Immunochemistry Technologies). Cells were resuspended in FACS buffer before analysis by a BD LSRII flow cytometer (BD Biosciences) or Attune (Life Technologies) and FlowJo software.

Cell sorting of resident renal phagocytes. Resident renal phagocytes $\left(\mathrm{CX}_{3} \mathrm{CR} 1^{+}\right.$cells) were isolated from $\mathrm{CX}_{3} C R 1^{\text {(gfp/+) }}$ mice using fluorescence cell sorting. The phenotype of $\mathrm{CX}_{3} \mathrm{CR} 1^{+}$cells was confirmed using intravital microscopy. Murine kidney tissue was isolated from mice after perfusion via saline injection into the heart. Kidney capsule and fat were removed before incubation of whole tissue in $2 \mathrm{mg} / \mathrm{ml}$ collagenase at $37^{\circ} \mathrm{C}$ for 30 minutes. Tissue homogenate was then passed through a $40-\mu \mathrm{M}$ nylon cell strainer and washed in HBSS. Viable cells were isolated using a density gradient by mixing cells with Percoll (GE Healthcare) and centrifuging at $800 g$ for 25 minutes. Cell viability was determined using SYTOX (Thermo Fisher Scientific) labeling. Cells were analyzed and sorted using a BD FACSARIA III (BD Biosciences).

\section{Kidney multiphoton intravital microscopy and quantification}

Mice were anesthetized using ketamine $(100 \mathrm{mg} / \mathrm{kg})$ and xylazine $(10 \mathrm{mg} / \mathrm{kg})$ supplemented with isoflurane before catheterization via tail vein for further administration of additional anaesthetic or labeling reagents. The kidney was exteriorized using a lateral incision and extended over the heated imaging platform. Imaging was done using a Leica multiphoton confocal microscope equipped with $\times 25$ water or $\times 63$ oil objective lens and Mai Tai pulsed infrared laser (Leica). Experiments were done at 1,000 $\mathrm{nM}$ excitation. Predefined settings for laser power and detector gain were used for all experiments. Time lapse imaging was acquired at 0.5 seconds per frame. $Z$-stacks were taken at 20- $\mu \mathrm{m}$ optical sections with 2- $\mu \mathrm{m}$ steps. Qtracker 655 (Thermo Fisher Scientific) was used in all mice to visualize capillaries, and SYTOX was used to label necrotic cells in vivo. Fluorescentconjugated DTA (Sigma-Aldrich) was synthesized using 1-ethyl-3[3-dimethylaminopropyl]carbodiimide hydrochloride crosslinked (EDC, Sigma-Aldrich) DTA with CF568-amine (Sigma-Aldrich). CF568 alone or CF568 conjugated to acetic acid was used as control. All acquisition and image processing was done with native LAS $\mathrm{X}$ software (Leica). Adherent LysM-GFP ${ }^{+}$and $\mathrm{CX}_{3} \mathrm{CR} 1-\mathrm{GFP}^{+}$cells were manually counted in each field based on their fixed position over the course of 2 minutes. Necrotic cells were quantified by measuring SYTOX-positive cells as a percentage of total cells (injury index) by Image J. A minimum of 3 fields were acquired and analyzed per mouse for all quantification. Renal dendritic cell activity in $C X_{3} C R 1^{(f f p /+)}$ mice was determined as previously described (36).

\section{Creatinine assays}

Renal function was determined in mouse serum using an enzymatic serum creatinine assay performed by Calgary Laboratory Services. 


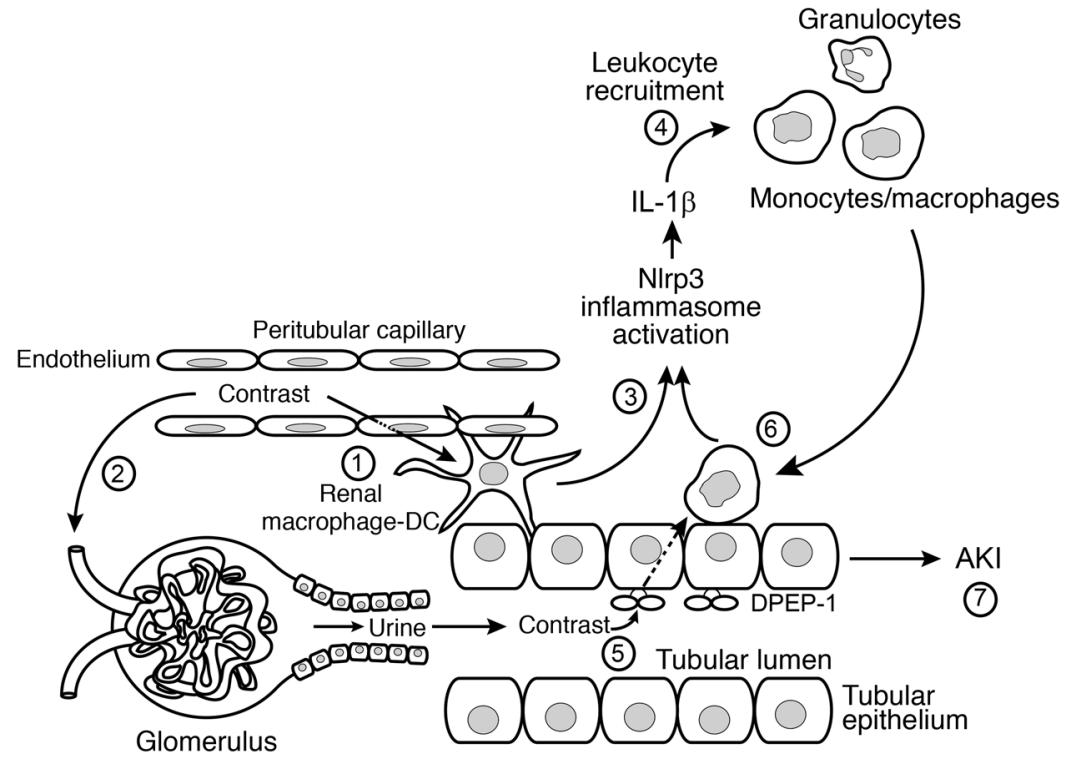

Figure 12. Schematic of CI-AKI. 1: Intravenous or intraarterial contrast agents enter the renal circulation and peritubular capillaries and are taken up by resident renal phagocytes. 2: Contrast is filtered at the glomerulus and enters the urine and renal tubule. In the hydrated state, contrast is rapidly excreted. 3: Contrast uptake by the resident renal phagocytes activates the NIrp3 inflammasome to generate IL-1 $1 \beta .4$ : IL-1 $\beta$ mediates leukocyte recruitment from the circulation into the kidney. 5: Contrast in the urine is taken up by the tubular cell by the brush border enzyme DPEP-1. The tubular uptake of contrast is enhanced in the volume-depleted state. 6: Recruited leukocytes ingest contrast transported from the urine through a direct interaction with tubular cells that further activates the inflammasome. 7: The activation of resident renal phagocytes, tubular uptake of contrast, and leukocyte recruitment are all necessary, but none alone, is sufficient to induce AKI.
Urinary creatinine in human specimens was measured using a Creatinine Companion Kit (Exocell) as per the manufacturer's instructions.

\section{Human contrast-induced acute kidney injury study}

Human urine specimens were obtained from an ongoing prospective biomarker study at the University of Calgary. Pre- and post-angiography urine specimens were chosen from 42 inpatients who underwent elective or urgent coronary angiogram procedures between December 2015 and May 2017 at the Foothills Medical Centre in Calgary, Alberta, Canada (Supplemental Table 1). Patients were eligible to participate in the study if they had evidence of kidney impairment (eGFR $<60 \mathrm{ml} / \mathrm{min} / 1.73 \mathrm{~m}^{2}$ ) and were willing to provide mid-stream urine samples. Individuals with prior renal transplantation, evidence of AKI in the previous 30 days ( $>26 \mu \mathrm{mol} / \mathrm{l}$ increase in serum creatinine in last 30 days), unable to produce a urine sample (ileal conduit or $<100 \mathrm{cc} / \mathrm{d}$ urine output), or residing outside of Alberta were not eligible to participate. Approximately $20-50 \mathrm{ml}$ urine was collected in a 90-ml container prior to coronary angiogram (baseline) and between 12 and 24 hours after coronary angiogram. Urine samples were set on ice and transported to the Biobank for the Molecular Classification of Kidney Disease (BMCKD, Snyder Institute for Chronic Diseases, Calgary, Alberta, Canada) within 1 hour. Specimens were immediately subjected to dipstick analysis (Chemstrip 10; Roche Diagnostics) and promptly centrifuged at $1,500 \mathrm{~g}$ for 10 minutes at $4^{\circ} \mathrm{C}$. The urine pellet as well as aliquots of the urine supernatant were placed in Micronic cryovials and stored at $-80^{\circ} \mathrm{C}$. Caspase -1 immunoblotting was performed on urinary pellet fractions. Meso Scale Discovery assays for KIM- 1 and IL-18 were performed on urine supernatants as per the manufacturer's protocol.

\section{Statistics}

Data are shown as mean \pm SEM. All experiments were performed at least 3 independent times. GraphPad Prism software version 7.03 was used to perform all statistical analyses. Results were analyzed for statistical variance using unpaired Student's $t$ test (2-tailed) or ANOVA with Tukey's or Dunnett's post hoc test where appropriate. Human urinary biomarker results were analyzed using Wilcoxon's signedrank test. Results at $P<0.05$ were considered statistically significant.

\section{Study approval}

Animal studies. All animal studies were reviewed and approved by the Animal Care Committee at the University of Calgary.

Human studies. All human studies were reviewed and approved by the Conjoint Health Research Ethics Board at the University of Calgary and Alberta Health Services (Calgary, Alberta, Canada). Patients provided written informed consent to participate in the CI-AKI study. HPTC isolation involved the secondary use of anonymized nephrectomy samples slated for disposal and therefore did not require patient consent.

\section{Author contributions}

AL performed the majority of experiments and wrote the manuscript. HJC, JMP, AUL, TK, SRC, JC, CFS, VN, BGJS, MCN, SLS, and PLB performed experiments, contributed data, and reviewed the manuscript. $\mathrm{HB}$ reviewed renal pathology. AMJ provided mouse strains, reagents, and expertise for necroptosis experiments and reviewed the manuscript. $\mathrm{MJH}$ provided expertise, analyzed $\mathrm{CX}_{3} \mathrm{CR} 1$ data, reviewed the manuscript, and supervised SLS. DLS provided expertise and DPEP-1 reagents, reviewed the manuscript, and supervised SRC. MTJ led the clinical CI-AKI study and provided patient data. JAM provided expertise, reviewed the manuscript, and supervised AUL. PK provided expertise, reporter mouse strains, and reagents; reviewed the manuscript; and supervised BGJS. CNJ provided intravital microscopy equipment and expertise, analyzed data, reviewed the manuscript, and supervised VN. DAM supervised and managed the entire project.

\section{Acknowledgments}

This work was supported by Operating and Foundation grants from the Canadian Institutes of Health Research (CIHR). Research was also supported by the Canadian National Trans- 
plantation Research Program (CNTRP) and the CIHR Inflammation in Chronic Disease Signature Initiative. DAM holds a Tier II Canada Research Chair. JAM was the holder of a Marie Skłodowska-Curie International Incoming Fellowship from the European Commission. MTJ is supported by a CIHR New Investigator Award. AL holds a Postdoctoral Scholarship Award from Alberta Innovates Health Solutions (AIHS). JMP and AL were also supported by Beverley Phillips Trainee Awards from the Snyder Institute for Chronic Disease, University of Calgary. The authors acknowledge May Ho and Stephen Robbins for their critical review of the manuscript, as well as Sharon A. Clark, Björn Petri, Kuo-Chieh Liao, and Adom Bondzi-Simpson for technical support. Infrastructure and technical support was provided by the Live Cell Imaging Facility, Mouse Phenomics Resource Laboratory, and the BMCKD at the Snyder Institute for Chronic Diseases.

Address correspondence to: Daniel Muruve, University of Calgary, 3280 Hospital Dr. NW, Calgary, AB, T2N 4Z6, Canada. Phone: 403.220.2418; Email: dmuruve@ucalgary.ca.
1. Azzalini L, Spagnoli V, Ly HQ. Contrast-induced nephropathy: from pathophysiology to preventive strategies. Can J Cardiol. 2016;32(2):247-255.

2. James MT, et al. Contrast-induced acute kidney injury and risk of adverse clinical outcomes after coronary angiography: a systematic review and meta-analysis. Circ Cardiovasc Interv. 2013;6(1):37-43.

3. McDonald JS, et al. Acute kidney injury after intravenous versus intra-arterial contrast material administration in a paired cohort. Invest Radiol. 2016;51(12):804-809.

4. Thomsen HS, Morcos SK. Risk of contrast-mediuminduced nephropathy in high-risk patients undergoing MDCT - a pooled analysis of two randomized trials. Eur Radiol.2009;19(4):891-897.

5. Tao SM, Wichmann JL, Schoepf UJ, Fuller SR, Lu GM, Zhang LJ. Contrast-induced nephropathy in CT: incidence, risk factors and strategies for prevention. Eur Radiol. 2016;26(9):3310-3318.

6. Cao Q, Wang Y, Harris DC. Pathogenic and protective role of macrophages in kidney disease. Am J Physiol Renal Physiol. 2013;305(1):F3-11.

7. Rogers NM, Ferenbach DA, Isenberg JS, Thomson AW, Hughes J. Dendritic cells and macrophages in the kidney: a spectrum of good and evil. Nat Rev Nephrol. 2014;10(11):625-643.

8. Kawakami T, et al. Resident renal mononuclear phagocytes comprise five discrete populations with distinct phenotypes and functions. J Immunol. 2013;191(6):3358-3372.

9. Stamatiades EG, et al. Immune Monitoring of trans-endothelial transport by kidney-resident macrophages. Cell. 2016;166(4):991-1003.

10. Yatim KM, Gosto M, Humar R, Williams AL, Oberbarnscheidt MH. Renal dendritic cells sample blood-borne antigen and guide T-cell migration to the kidney by means of intravascular processes. Kidney Int. 2016;90(4):818-827.

11. Chung $\mathrm{H}$, et al. NLRP3 regulates a non-canonical platform for caspase-8 activation during epithelial cell apoptosis. Cell Death Differ. 2016;23(8):1331-1346.

12. Iyer SS, et al. Necrotic cells trigger a sterile inflammatory response through the Nlrp3 inflammasome. Proc Natl Acad Sci U S A. 2009;106(48):20388-20393.
13. Shigeoka AA, et al. An inflammasomeindependent role for epithelial-expressed Nlrp3 in renal ischemia-reperfusion injury. J Immunol. 2010;185(10):6277-6285.

14. Vilaysane A, et al. The NLRP3 inflammasome promotes renal inflammation and contributes to CKD. J Am Soc Nephrol. 2010;21(10):1732-1744.

15. Mulay SR, et al. Calcium oxalate crystals induce renal inflammation by NLRP3-mediated IL-1 $\beta$ secretion. J Clin Invest. 2013;123(1):236-246.

16. Ludwig-Portugall I, et al. An NLRP3-specific inflammasome inhibitor attenuates crystalinduced kidney fibrosis in mice. Kidney Int. 2016;90(3):525-539.

17. Faust N, Varas F, Kelly LM, Heck S, Graf T. Insertion of enhanced green fluorescent protein into the lysozyme gene creates mice with green fluorescent granulocytes and macrophages. Blood. 2000;96(2):719-726.

18. Haller C, Hizoh I. The cytotoxicity of iodinated radiocontrast agents on renal cells in vitro. Invest Radiol. 2004;39(3):149-154.

19. Linkermann A, et al. The RIP1-kinase inhibitor necrostatin-1 prevents osmotic nephrosis and contrast-induced AKI in mice. JAm Soc Nephrol. 2013;24(10):1545-1557.

20. Lawlor KE, et al. RIPK3 promotes cell death and NLRP3 inflammasome activation in the absence of MLKL. Nat Commun. 2015;6:6282.

21. Neyen C, et al. Macrophage scavenger receptor A mediates adhesion to apolipoproteins A-I and E. Biochemistry. 2009;48(50):11858-11871.

22. Navab M, et al. Oral administration of an Apo A-I mimetic Peptide synthesized from D-amino acids dramatically reduces atherosclerosis in mice independent of plasma cholesterol. Circulation. 2002;105(3):290-292.

23. Hochweller K, Striegler J, Hämmerling GJ, Garbi N. A novel CD11c.DTR transgenic mouse for depletion of dendritic cells reveals their requirement for homeostatic proliferation of natural killer cells. Eur JImmunol. 2008;38(10):2776-2783.

24. Camano S, et al. Cilastatin attenuates cisplatin-induced proximal tubular cell damage. JPharmacol Exp Ther. 2010;334(2):419-429.

25. Humanes B, et al. Protective effects of cilastatin against vancomycin-induced nephrotoxicity.
Biomed Res Int. 2015;2015:704382.

26. Hori Y, et al. Megalin blockade with cilastatin suppresses drug-induced nephrotoxicity. J Am Soc Nephrol. 2017;28(6):1783-1791.

27. Pang S, Urquhart P, Hooper NM. N-glycans, not the GPI anchor, mediate the apical targeting of a naturally glycosylated, GPI-anchored protein in polarised epithelial cells. J Cell Sci. 2004;117(Pt 21):5079-5086.

28. Navaneethan SD, Singh S, Appasamy S, Wing RE, Sehgal AR. Sodium bicarbonate therapy for prevention of contrast-induced nephropathy: a systematic review and meta-analysis. Am J Kidney Dis. 2009;53(4):617-627.

29. Solomon R, Werner C, Mann D, D'Elia J, Silva P. Effects of saline, mannitol, and furosemide to prevent acute decreases in renal function induced by radiocontrast agents. $N$ Engl J Med. 1994;331(21):1416-1420.

30. Ip WK, Medzhitov R. Macrophages monitor tissue osmolarity and induce inflammatory response through NLRP3 and NLRC4 inflammasome activation. Nat Commun. 2015;6:6931.

31. Tschopp J, Schroder K. NLRP3 inflammasome activation: The convergence of multiple signalling pathways on ROS production? Nat Rev Immunol. 2010;10(3):210-215.

32. Bakker PJ, et al. A tissue-specific role for Nlrp3 in tubular epithelial repair after renal ischemia/ reperfusion. Am J Pathol. 2014;184(7):2013-2022.

33. Shen J, et al. NLRP3 inflammasome mediates contrast media-induced acute kidney injury by regulating cell apoptosis. Sci Rep. 2016;6:34682.

34. Kahan FM, Kropp H, Sundelof JG, Birnbaum J. Thienamycin: development of imipenen-cilastatin. JAntimicrob Chemother. 1983;12 Suppl D:1-35.

35. Lin SL, Kisseleva T, Brenner DA, Duffield JS. Pericytes and perivascular fibroblasts are the primary source of collagen-producing cells in obstructive fibrosis of the kidney. Am J Pathol. 2008;173(6):1617-1627.

36. Snelgrove SL, et al. Renal dendritic cells adopt a pro-inflammatory phenotype in obstructive uropathy to activate $\mathrm{T}$ cells but do not directly contribute to fibrosis. Am J Pathol. 2012;180(1):91-103. 